\title{
Raman Spectra of Methane, Ethylene, Ethane, Dimethyl ether, Formaldehyde and Propane for Combustion Applications
}

\author{
G. Magnotti ${ }^{\mathrm{a}}$, U. KC ${ }^{\mathrm{b}, \mathrm{c}}$, P. L. Varghese ${ }^{\mathrm{c}}$, R.S. Barlow ${ }^{\mathrm{a}}$ \\ ${ }^{a}$ Combustion Research Facility, \\ Sandia National Laboratories, Livermore CA 94550, USA \\ ${ }^{b}$ Clean Combustion Research Center, \\ King Abdullah University of Science and Technology, Thuwal 23955, Saudi Arabia \\ ${ }^{c}$ Department of Aerospace Engineering and Engineering Mechanics, \\ The University of Texas at Austin, Austin, TX 78712, USA
}

\begin{abstract}
Spontaneous Raman scattering measurements of temperature and major species concentration in hydrocarbon-air flames require detailed knowledge of the Raman spectra of the hydrocarbons present when fuels more complex than methane are used. Although hydrocarbon spectra have been extensively studied at room temperature, there are no data available at higher temperatures. Quantum mechanical calculations, when available are not sufficiently accurate for combustion applications. This work presents experimental measurements of spontaneous Stokes-Raman scattering spectra of methane, ethylene, ethane, dimethyl ether, formaldehyde and propane in the temperature range 300-860 K. Raman spectra from heated hydrocarbons jets have been collected with a higher resolution than is generally employed for Raman measurements in combustion applications. A set of synthetic spectra have been generated for each hydrocarbon, providing the basis for extrapolation to higher temperatures. The spectra provided here will enable simultaneous measurements of multiple hydrocarbons in flames. This capability will greatly extend the range of applicability of Raman measurements in combustion applications. In addition, the experimental spectra provide a validation dataset for quantum mechanical models.
\end{abstract}

Keywords: Raman Spectroscopy, Hydrocarbons, Combustion

Corresponding Author: Gaetano Magnotti, email: gmagnot@sandia.gov

\section{Introduction}

Raman/Rayleigh scattering is a powerful laser diagnostic technique for measurements of temperature and species concentrations in combustion environments.[1-5] The line-imaging Raman/Rayleigh scattering instrument at Sandia National Laboratories (SNL) provides instantaneous measurements of temperature and major species concentrations $\left(\mathrm{N}_{2}, \mathrm{O}_{2}, \mathrm{CO}_{2}, \mathrm{CO}\right.$, $\mathrm{H}_{2} \mathrm{O}, \mathrm{H}_{2}$ and $\mathrm{CH}_{4}$ ) in flames, over a $6 \mathrm{~mm}$ probe line, with a spatial resolution of $\sim 100$ microns. The instrument is routinely applied to measurements in laboratory-scale flames that are specifically designed to advance the understanding of the turbulence-chemistry interaction, and to provide validation databases for computational models. Recent measurement campaigns focused on the effects of preferential diffusion, fuel stratification, and swirl in methane-air flames. [6-8] The combustion community has a strong interest in extending the range of applicability of this instrument to fuels more complex than methane. However, the data 
processing to extract species concentrations and temperature from Raman/Rayleigh measurements is more challenging for complex fuels, particularly when simultaneous measurements of multiple hydrocarbons are needed.

Recently Fuest et al. [9] introduced the "hybrid method" to extract concentration measurements from line imaging Raman data. The hybrid method combines the benefits of the two previously dominant approaches, the "matrix inversion" [10] and the spectral fitting approach [11]. In the matrix inversion approach "Raman channels" are defined on the CCD camera by on-chip binning over assigned spectral regions. The resulting Raman signal vector $S$ (at given location $x$ and instantaneous shot) is related to the species number density vector $N$ through the matrix $C$ :

$$
S=C(T) \cdot N
$$

The signal on each channel contains the Raman response of its associated species (diagonal terms of the $C$-matrix), and contributions (crosstalk) from all the other species (off diagonal terms of the $C$-matrix). Many crosstalk contributions are negligible, making the $C$ matrix sparse. The matrix elements are functions of temperature and are obtained by empirical calibration. Given the signal vector $S$ and the Raman response matrix $C$, the number densities can be obtained by matrix inversion. The process is iterative since the matrix $C$ depends on the temperature that is unknown. A guess temperature is estimated from the Rayleigh signal, the number densities are computed, and a more accurate temperature is obtained using the computed Rayleigh cross-section. This process is iterated until the temperature converges. Problems arise in 1D Raman imaging for turbulent combustion applications. Beam steering caused by steep density gradients is responsible for shifts of the entire Raman spectra with respect to the fixed Raman channels. Further complications arise from the "bowing effect", an intrinsic feature of compact on-axis spectrometers such as the one used in our experimental set-up (details in Section II).[12] The image of the entrance slit is distorted by the diffraction grating, causing a red shift of up to $1.2 \mathrm{~nm}$ between the bowing center and the edge of the measurement line. The Raman channels therefore cover a slightly different spectral region, at different locations along the probe line. Because of beam steering and the bowing effect, the elements of the matrix $C$ are also a function of the spatial position along the probe line, and of the instantaneous laser pointing.

An alternative to the matrix inversion approach is direct spectral fitting of the Raman data to a theoretical or empirical library. This approach preserves the spectral information and can correct for beam steering and bowing effect by shifting the spectra during the fitting procedure. Moreover, spectral fitting allows for a better treatment of the background by fitting it simultaneously with the spectra itself. The drawback of the approach is a significantly lower signal-to-noise ratio with consequent lower precision than what can be achieved by on-chip binning due to reduced readout noise. In addition the data acquisition rate is reduced, and the spectra are sensitive to the lineshape and to the instrument function. In the hybrid approach the elements of the matrix $C$ are computed by integrating the theoretical Raman spectra over the Raman channels for different values of the temperature, position along the probe line, and pixel shift due to beam steering. This approach allows automatic compensation for spectral shift due to beam steering and the bowing effect, while preserving the higher precision allowed by on-chip binning. 
The hybrid approach requires the knowledge of the Raman spectra as functions of the temperature. In previous Raman measurements in methane-air flames performed at Sandia, the spectra of all the major species, except methane, were obtained from quantum mechanical calculations from the Ramses algorithm [11]. Since methane Raman spectra at elevated temperatures are not available in Ramses, the elements of the matrix $C$ associated with the contributions from methane are assumed independent of the location along the probe, and of the pixel shift, (as in the regular matrix inversion approach). The temperature dependence was determined empirically from measurements in a stable, laminar flame from a Tsuji burner [13]. The strongest Raman signal from methane is due to the $\mathrm{CH}$ vibrational stretch and it is in the $2600-3300 \mathrm{~cm}^{-1}$ range, a region well separated from the channels associated with other major combustion species. By choosing a sufficiently large methane Raman channel, the matrix elements associated with the methane channel are independent of the pixel shift and of the position along the probe line. A weaker Raman signal from methane is located in the 1200-1900 $\mathrm{cm}^{-1}$ range resulting from a $\mathrm{CH}_{2}$ bending mode, causing a crosstalk onto the $\mathrm{CO}_{2}$ (1133-1489 $\left.\mathrm{cm}^{-1}\right)$ and $\mathrm{O}_{2}\left(1489-1662 \mathrm{~cm}^{-1}\right)$ Raman channels. The methane Raman signals in the $\mathrm{CO}_{2}$ and $\mathrm{O}_{2}$ channels are sensitive to the bowing effect, with $\sim 10 \%$ changes in the integrated Raman scattering intensity between the center and the edge of the $6 \mathrm{~mm}$ probe line. Since the methane crosstalk is an order of magnitude weaker than the $\mathrm{CO}_{2}$ and $\mathrm{O}_{2}$ Raman signal, the error in the concentration caused by neglecting the bowing effect is less than $1 \%$ for typical methane-air flame compositions.

The approach used to determine species concentrations for methane can be extended to Raman measurements in flames where the fuel is a single hydrocarbon, defining a region associated with the strong $v_{1} \mathrm{CH}$-stretch transition to evaluate the hydrocarbon concentrations from that, and determining the temperature dependence empirically. Neglecting the beam steering and the bowing effect will produce an error in the $\mathrm{O}_{2}$ and $\mathrm{CO}_{2}$ concentrations due to crosstalk, but it will not have a significant effect on the hydrocarbon concentration or the temperature. The approach breaks down for more complex fuels where multiple hydrocarbons are present simultaneously. This is a case of great interest for combustion applications, because it is closer to what is encountered in a practical combustor. For example, typical composition of natural gas includes a significant percentage of ethane and propane, in addition to methane. Mixtures of methane and ethylene are often used as surrogate of cracked JP-7 fuel for aerospace applications.[14] Even when the fuel is a single hydrocarbon, combustion intermediates concentrations can be significant, and cannot be neglected without causing large errors in the temperature measurements. This is the case for instance in DME-air flames where the combined mole fractions of methane, formaldehyde, acetylene, ethylene, ethane (combustion intermediates) reach $8 \%$ of the total mole fractions.[15]

Figure 1a) shows the room temperature spectra of methane, ethylene, ethane, and DME, obtained with the spectrometer of Ref. [6] that has a 1200 lines/mm grating. Raman spectra in the $\mathrm{CH}$-stretch region are closely overlapped giving rise to very large crosstalk. The vertical lines define a plausible channel for methane $\left(2882-2949 \mathrm{~cm}^{-1}\right)$, with boundaries defined by the frequencies where the methane Raman signal is $10 \%$ of its peak value at $300 \mathrm{~K}$. At room temperature, assuming equal concentrations of all hydrocarbons, the integrated Raman signal of DME and ethane on the methane channel (crosstalks of DME and ethane onto methane) are $61 \%$ and $127 \%$ of the integrated methane Raman signal. Changes in the crosstalks due to the bowing effect are up to 5\% and 20\% for DME and ethane, respectively, and cannot be neglected without large errors in the concentration measurements, especially when measuring combustion 
intermediates. Figure 1b) shows the same spectra as the upper plot, obtained with the same optical configuration but with a higher dispersion grating (4165 lines/mm). The methane Raman channel boundaries are again chosen to correspond to $10 \%$ of the peak value in the high dispersion spectrum, but this corresponds to a much narrower spectral interval $\left(2911-2925 \mathrm{~cm}^{-1}\right)$. Consequently, the overlap is greatly reduced, and the crosstalk of DME and ethane onto the methane channel $\left(2911-2925 \mathrm{~cm}^{-1}\right)$, are only $17 \%$ and $10 \%$ of the integrated $\mathrm{CH}_{4}$ signal, a reduction by a factor of 4 and 12, respectively. The major drawback of using a higher dispersion is that a smaller spectral region is imaged on the CCD camera, making it inadequate for Raman measurements of the other combustion species. The issue can be overcome by a dual-resolution Raman instrument, imaging simultaneously the $800-4600 \mathrm{~cm}^{-1}$ region at the resolution of Fig. 1a) and the $2700-3280 \mathrm{~cm}^{-1}$ region with the dispersion of Fig 1b), using two detection systems. Such an instrument, combined with the hybrid approach to correct for beam steering and for the bowing effect has potential for allowing Raman measurements of multiple hydrocarbons in combustion environments. Recently, we developed a polarization-separation Raman instrument that simultaneously acquires two orthogonal polarization components of the Raman spectra to remove fluorescence interference in rich hydrocarbon flames.[16] The instrument can be modified by using a higher dispersion grating in one of the spectrometers, so that dual-resolution Raman spectra can be collected.

The focus of this paper is the development of a library of Raman spectra for methane, ethane, ethylene, DME, and propane, to enable the hybrid approach for the analysis of Raman data acquired with a dual-resolution instrument. Although ambient temperature Raman spectra of all these hydrocarbons are available in the open literature, high temperature measurements are available only for methane [17]. Theoretical models in the temperature range useful for combustion applications have been proposed only recently for methane [17], but they are too complex for heavier hydrocarbons. Ekoto et al.[18] reported a set of experimental Raman spectra for the aforementioned hydrocarbons in the 300-900 K range, obtained with the low dispersion spectrometer, and therefore inadequate for the dual-resolution instrument. The spectra presented in this paper cover the same temperature range of Ref. [18], but are obtained with a grating of 4165 lines/mm (for $0.03 \mathrm{~nm} /$ pixel dispersion) and a slit width of $100 \mu \mathrm{m}$, resulting in a higher spectral resolution. We collected two sets of spectra using an appropriately oriented thin film polarizer. The sets labelled "perpendicular polarization" and "parallel polarization" contain, respectively, the component of the Raman spectra with polarization perpendicular and parallel to the scatter plane spanned by the direction of the incident radiation and the direction of observation, which is 90 degrees in the current configuration. The exciting laser is perpendicularly polarized with respect to the scatter plane. Figure 2c) shows the orientation of the laser polarization and of the scatter plane. A third set of spectra, labelled "no polarizer" was obtained with no thin film polarizer in the path. Because of the large signal collection angle the "parallel" component includes also contributions from the isotropic component of the Raman signal. Hence the depolarization ratio, defined as the ratio of the parallel and perpendicular component, is always larger than predicted from theory assuming very small collection solid angle [19]. Synthetic spectra obtained as sum of Gaussian curves, with width and amplitude of the Gaussian curves expressed as a function of the temperature were generated by a fitting algorithm, and compared to the experimental spectra. The number of Gaussian curves is determined by the algorithm, and it is not representative of individual transitions or branches. The synthetic spectra can later on be convolved with the appropriate apparatus function to generate spectra matching the instrument resolution (different for low and high resolution sides 
of the dual-resolution system), and then integrated over the designed spectral region to obtain the $C$ matrix. The last section describes a strategy to extend the synthetic spectra to higher temperatures, and demonstrates its application to methane spectra.

\section{Experimental set-up}

The main components of the experimental set-up used in this work are an electric heater to control the gas mixture temperature, a low resolution Raman/Rayleigh instrument to accurately determine the temperature dependence of the integrated Raman signal, and a high resolution Raman spectrometer to provide detailed spectral information. Figure 2 shows a schematic and a photograph of the heater. It consists of a $0.635 \mathrm{~m}$ long quartz tube, with a $30.1 \mathrm{~mm}$ outer diameter at the exit and a $1.5 \mathrm{~mm}$ wall thickness. The test gases are injected in the quartz tube by two shorter quartz tubes placed $0.51 \mathrm{~m}$ from the flanged base. The low resolution Raman data were collected in a mixture of $\mathrm{N}_{2}$ and the chosen hydrocarbon, with total flow rates of $\sim 50$ SLM. The mixture ratio was selected to maximize the Raman signal at room temperature without saturating the camera. The high resolution measurements were made in pure hydrocarbons (Matheson C.P. grade) at flow rates of $~ 10$ SLM. Mass flow rates were controlled by MKS flow controller. The gas temperature near the exit is monitored by a type $\mathrm{K}$ thermocouple, inserted into a port $0.457 \mathrm{~m}$ from the flanged base. A silicon carbide heater element, $32 \mathrm{~cm}$ long with a heated length of $21 \mathrm{~cm}$ was seated against the flanged quartz base with a clamp designed to minimize mechanical stress on the quartz body. A 2 inch thick fiberglass insulation blanket surrounds the heater to minimize heat losses. A Variac variable transformer provides power to the heater, and the supplied current was manually adjusted to achieve the desired thermocouple temperature. Gas temperatures are limited to approximately $900 \mathrm{~K}$ at the heater exit. Higher temperatures cause outgassing from the heater element that interferes with the Raman measurements. Pyrolysis also becomes a concern at high temperature because the temperature next to the heating element is higher than what is measured by the thermocouple resulting in pyrolitic decomposition of the hydrocarbon. This is particularly evident for DME, where methane and formaldehyde spectra appear for thermocouple temperatures above $700 \mathrm{~K}$. Raman measurements were taken $25 \mathrm{~mm}$ from the surface, sufficiently close to the exit so that no air is entrained, but distant enough to avoid scattering from the surface. Black barriers visible in Fig. 2 were used to minimize backscatter from the incident laser light. The energy of the laser was adjusted to maximize signal to noise ratio, and it is limited by the photo-fragmentation threshold of each hydrocarbon. Photo-fragmentation is typically associated with formation of $\mathrm{C}_{2}$ that produces strong fluorescence interference peaks, easily detectable in the low resolution spectra.

The "low resolution" Raman/Rayleigh instrument was the same as described in [20], and routinely used for measurements in $\mathrm{CH}_{4}$-air flames. The instrument combines Rayleigh and Raman scattering for single-shot acquisition of temperature and major species concentration $\left(\mathrm{N}_{2}\right.$, $\mathrm{O}_{2}, \mathrm{CO}, \mathrm{CO}_{2}, \mathrm{CH}_{4}, \mathrm{H}_{2}, \mathrm{H}_{2} \mathrm{O}$ ) along a $6 \mathrm{~mm}$ probe line. The Raman-Rayleigh imaging probe is provided by four frequency doubled Nd:YAG lasers, with $100 \mathrm{~ns}$ delay between each pulse, and sent through three optical delay lines, to generate a $1.4 \mathrm{~J} /$ pulse combined laser beam, temporally stretched over $400 \mathrm{~ns}$ to prevent optical breakdown. A pair of $150 \mathrm{~mm}$ diameter achromatic lenses (Linos Photonics f/2 and f/4) focuses the Raman/Rayleigh signal into the detection system. The main internal components include two custom-built motor-driven chopper wheels, six commercial camera lenses, a custom transmission grating (1200 lines/mm Kaiser Optical) and mirrors and filter to separate the signal from Raman scattering ( 550-700 nm) and Rayleigh 
scattering (532 nm). A Princeton Instruments 1340/400 EMB CCD camera is used for the Rayleigh signal. A non-intensified, low-noise, cryogenically cooled CCD camera (Princeton Instruments VersArray 1300B with CryoTiger cooling unit, $-110^{\circ} \mathrm{C}$ operating temperature) is used for Raman detection. Gating for the Raman camera is provided by a rotating shutter operating at $21000 \mathrm{rpm}$. The stationary and rotating slits are $0.50 \mathrm{~mm}$ and $0.55 \mathrm{~mm}$, respectively, and they are centered at a radius of $63.5 \mathrm{~mm}$, producing a gate width of $3.9 \mu \mathrm{s}$ (FWHM). This high-speed shutter is operated in tandem with a low-speed (3000 rpm) shutter, placed at the focal plane of the main collection lens, which provides $300 \mu \mathrm{s}$ gating for the Rayleigh camera. The two wheels are controlled by a custom-made phase-locked loop (PLL) circuit, which also provides the master timing signals for operation of lasers and cameras. The Raman camera is binned spatially providing 100 microns spatial resolution. Raman channels for all major species are defined in the spectral direction and the Raman signal is hardware binned in this direction. Two channels are defined for the hydrocarbons, one covering the $882-1791 \mathrm{~cm}^{-1}$ Raman shift region, the other covering the $2548-3434 \mathrm{~cm}^{-1}$ region. The low resolution Raman/Rayleigh system is not used to generate the Raman spectra, but to provide accurate temperature dependence of the integrated signal.

The Raman spectra shown in this paper were obtained using a "high resolution" Raman detection system placed opposite to the "low resolution" system described above. A similar version of the spectrograph has been previously used by $\mathrm{KC}$ et al. [21] to acquire high resolution $\mathrm{N}_{2}$ spectra in laminar flames. A pair of $150 \mathrm{~mm}$ diameter achromatic lenses (Linos Photonics $\mathrm{f} / 2$ and f/4), identical to the ones used for the "low resolution" system, focused the Raman signal at the entrance of a Holospec $\mathrm{f} / 1.8 \mathrm{i}$ spectrograph (Kaiser Optical Instruments). The Raman/Rayleigh signal was then collimated by a camera lens, the Rayleigh signal was removed by a holographic notch filter with an OD $>6$ at $532 \mathrm{~nm}$ and a FWHM transmission width $<350$ $\mathrm{cm}^{-1}$. The remaining Raman signal was then focused by a second lens on a $100 \mu \mathrm{m}$ slit and collimated by a subsequent camera lens onto the grating. Two holographic transmission gratings, with 4165 lines/mm, were available to disperse the Raman signal. The HDG-573 grating was used to probe the hydrocarbon bending modes in the $882-1791 \mathrm{~cm}^{-1}$ region; the HDG 631 grating was used to probe the $\mathrm{CH}$ stretching modes in the $2548-3434 \mathrm{~cm}^{-1}$ region. The dispersed light was focused into a Princeton Instrument LN cooled Versarray camera. The CCD array was horizontally binned over 90 pixels, corresponding to a measurement volume of $\sim 1 \mathrm{~mm}$ to improve the signal to noise ratio. No binning was applied in the spectral direction and a spectral dispersion of $\sim 0.02 \mathrm{~nm} /$ pixel was achieved. The detection assembly was enclosed within black curtains and all additional light sources were carefully removed to reduce scattering from ambient light.

\section{Temperature dependence of the integrated Raman signal}

This section discusses the temperature dependence of the integrated Raman signal collected with the "low resolution" Raman/Rayleigh system. This approach is preferred to direct integration from the "high resolution" spectra, because of higher signal to noise ratio, reduced sensitivity to changes in the background, and more accurate temperature measurements. The integration width and resulting binning regions for the hydrocarbons were chosen to match the regions covered by the two gratings used for the high resolution spectrometer, covering the 882$1791 \mathrm{~cm}^{-1}\left(\mathrm{CH}_{2}\right.$-bend region) and the $2548-3434 \mathrm{~cm}^{-1}$ region $(\mathrm{CH}$-stretch region). We collected Raman/Rayleigh data in mixtures of $\mathrm{N}_{2}$ and one hydrocarbon; the laser energy and the ratio of 
the hydrocarbon to the $\mathrm{N}_{2}$ were optimized to avoid breakdown and photo-fragmentation, and to obtain a signal of $~ 50000$ counts in the $\mathrm{CH}$-stretch bin, at room temperature. The laser energy and the integrated $\mathrm{N}_{2}$ to hydrocarbon ratio were kept constant while varying the gas temperature. Temperature was measured by Rayleigh scattering, assuming that the mixture Rayleigh cross section is temperature independent over the range of the experiments. The thermocouple measurements, taken inside the quartz tube, $0.178 \mathrm{~m}$ from the exit, were systematically higher than the Rayleigh measurements, and a calibration curve was derived to correct the thermocouple readings and facilitate accurate control of the heater. The Raman signal was background subtracted, multiplied by a calibration curve that removes the effects of the non-uniform throughput in the spatial direction, and averaged over the central $1 \mathrm{~mm}$ and over 500 single shots. The calibration curve was obtained by previous measurements in a uniform mixture of nitrogen and each hydrocarbon. The temperature dependent Raman signal in each channel was then normalized by its value at the ambient temperature. To remove the density dependence, the Raman signal was divided by the ratio between the Rayleigh signal at given temperature and the Rayleigh signal at ambient temperature.

Raman/Rayleigh measurements taken at room temperature in pure hydrocarbons and pure $\mathrm{N}_{2}$ jets provide the Rayleigh cross section relative to $\mathrm{N}_{2}$ and the ratios of the hydrocarbon Raman signal in the $\mathrm{CH}_{2}$ - bend and $\mathrm{CH}$-stretch regions to the $\mathrm{N}_{2}$ Raman signal (2208-2429 $\mathrm{cm}^{-1}$ ) taken without a polarizer. The integrated Raman signals in $\mathrm{N}_{2}$ with the perpendicular and parallel polarizer orientations are $81.5 \%$ and $9.5 \%$ of the signal collected with no polarizer, respectively. The results are summarized in Table I. The integrated signal in the $\mathrm{CH}$-stretch region is between 10 and 26 times stronger in the hydrocarbon than in nitrogen, allowing for higher detection limits than other combustion species. The Raman signal in the $\mathrm{CH}_{2}$-bend region is weak for methane ( 0.6 of the $\mathrm{N}_{2}$ signal), but 3 to 5.5 times larger for the other hydrocarbons. This makes combustion measurements particularly challenging because the crosstalk of the hydrocarbons onto the $\mathrm{CO}_{2}$ and $\mathrm{O}_{2}$ channels can be comparable to the $\mathrm{CO}_{2}$ and $\mathrm{O}_{2}$ contributions. As an example for an unstrained $\mathrm{C}_{2} \mathrm{H}_{4}$-air flame with equivalence ratio of $0.7, \mathrm{C}_{2} \mathrm{H}_{4}$ mole fraction is 5.5 times smaller than $\mathrm{O}_{2}$, producing a crosstalk contribution that is greater than $20 \%$ of the $\mathrm{O}_{2}$ signal, and accounts for most of the signal in the $\mathrm{CO}_{2}$ channel. In higher temperature regions the relative contribution on the $\mathrm{O}_{2}$ channel decreases (for lean flames), but the contribution of $\mathrm{C}_{2} \mathrm{H}_{4}$ onto the $\mathrm{CO}_{2}$ channel is higher than the $\mathrm{CO}_{2}$ signal up to $1100 \mathrm{~K}$.

The symbols in Fig. 3 show the density-independent, normalized, integrated Raman signal as function of the Rayleigh temperature for the $\mathrm{CH}$-stretch hydrocarbon channel (on the right) and for the $\mathrm{CH}_{2}$-bend channel (on the left). The error bars represent \pm one standard deviation, and are typically $\sim 1 \%$ of the mean value. The integrated ro-vibrational $\mathrm{N}_{2}$ scattering signal (2208-2429 $\mathrm{cm}^{-1}$ ) acquired with the perpendicular polarizer is also shown in the methane plot and compared to Ramses calculations computed with the appropriate polarization. The measured $\mathrm{N}_{2}$ Raman signal is within $0.5 \%$ of the Ramses predicted value and demonstrates the accuracy of the measurements. The continuous curves are third order polynomials obtained by a least-squares fit to the experimental results.

The component of the spectrum with polarization parallel to the scattering plane has a weaker dependence on temperature than the perpendicular portion. With the exception of $\mathrm{CH}_{4}$, changes in the normalized Raman signal with temperature are much stronger in the $\mathrm{CH}_{2}$-bend region. Fluorescence of $\mathrm{C}_{2}$, obtained by photo-fragmentation at higher temperature, produces a large interference signal in the $\mathrm{CH}_{2}$-bend and in the $\mathrm{CO}$ region. The $\mathrm{C}_{2}$ fluorescence could not be responsible for the observed temperature dependence in the $\mathrm{CH}_{2}$-bend region because we 
observed no changes in the $\mathrm{CO}$ channel. Detailed comments on the temperature dependence for each hydrocarbon can be found in the next section together with descriptions of the spectra.

\section{High resolution hydrocarbon spectra}

The spectra shown in this section were collected in heated hydrocarbon jets with no $\mathrm{N}_{2}$ dilution, using the high resolution spectrometer previously described. $\mathrm{N}_{2}$ dilution allows higher laser energies, but the net effect is a reduction of the hydrocarbon Raman signal because of the lower concentration. The laser energy was tuned for the different hydrocarbons and temperatures in order to achieve the maximum Raman signal without inducing breakdown or photofragmentation. Occasional spectra containing evidence of photo-fragmentation and of laser induced breakdown were removed during the data processing. A quadratic polynomial that provides the conversion from camera pixel to wavelength was obtained by matching the measured pixel locations of spectral peaks from a neon spectral lamp to their expected wavelength from the lamp user manual. Background spectra were obtained by averaging 100 instantaneous spectra taken with the laser blocked. A throughput calibration curve is needed to correct for the frequency-dependent transmissivity of the grating and of other optical components. A standard L-931 tungsten lamp provided the calibration source. The spectral irradiance of this source is tabulated in the product manual. The tabulated data did not cover our spectral region of interest, $(560-590 \mathrm{~nm}, 615-650 \mathrm{~nm})$, and the available data were fitted to a modified Planck function to obtain the irradiance in $\mathrm{W} / \mathrm{m}^{2} / \mathrm{nm}$ :

$$
I=3.741770 \cdot 10^{20} \cdot m_{1} \cdot\left(1+m_{2} \lambda\right) /\left(\lambda^{5} \cdot \exp \left(\left(1.4388 \cdot \frac{10^{-7}}{\lambda T}\right)-1\right)\right)
$$

where $T$ is the estimated temperature of the tungsten filament $(2851 \mathrm{~K})$ and $m_{1}$ and $m_{2}$ are the best fit coefficients. The irradiance was then multiplied by the dispersion (derivative of the pixel to $\mathrm{cm}^{-1}$ conversion curve from the neon lamp measurements) to obtain the irradiance per pixel. The calibration curve was obtained as the ratio between the expected and the measured irradiance per pixel. The raw data were background subtracted, normalized by the laser energy, multiplied by the throughput calibration curve and averaged over the total number of realizations. The average spectra were normalized so that the integrated signal matches the hydrocarbon/ $\mathrm{N}_{2}$ ratio reported in Table 1 . The temperature dependence of the high resolution spectra was corrected by the third order polynomials of Fig. 3 to make use of the higher accuracy of the low resolution instrument. The resulting spectra in Fig.4 to Fig. 19 are plotted as a function of Raman shift. Note that the high resolution spectra reported here are averaged over \pm 45 pixels around the bowing center, so that the bowing effect is smaller than 1 pixel. The Raman shift was obtained as the difference between the Nd:YAG laser frequency and the frequency of the collected Raman signal. The value of the Nd:YAG laser frequency was not measured, but set to $18793.2 \mathrm{~cm}^{-1}$ so that the methane $v_{1}$ peak falls at $2917 \mathrm{~cm}^{-1}$.

The following subsections describe the spectra collected for each hydrocarbon. The spectra are provided in graphical form in Figs. 4-18 and in tabular form in the supplemental material. The temperature dependence of the spectra, the polarization dependence and several spectral features are discussed with particular attention to combustion applications. The spectra provided here can also be used to validate theoretical models. As a general rule an $\mathrm{N}$-atomic molecule with 
a non-linear structure has $3 N-6$ normal vibrational modes. Fundamental vibrational frequencies for all the hydrocarbons investigated in this study are listed in [22].The general harmonic oscillator selection rule is $\Delta \mathrm{v}_{\mathrm{i}}= \pm 1$, where $\mathrm{v}$ is the vibrational quantum number, but to be Raman active a change in the induced dipole moment is required, imposing more restrictive selection rules that depend on the symmetry properties of the molecule. Most of the transitions discussed in the section below are fundamental transition $\mathrm{v}=0 \rightarrow 1$ but at higher temperatures, where higher vibrational energy states are populated, hot band transitions such as $v=1 \rightarrow 2$ and $v=2 \rightarrow 3$ can be observed. First overtone modes $\mathrm{v}=0 \rightarrow 2$, although generally weaker are also observed. Higher order overtones are too weak to be detected and can be neglected for combustion applications. Combination modes occur when two or more fundamental frequencies interact with each other; this often involves modes that are not Raman active at their fundamental frequency. Overtones and combination modes are typically much weaker than the fundamental modes, but their amplitude can be strongly amplified by resonances like the Fermi resonance. When a fundamental and an overtone or combination mode have approximately the same frequency, the two modes mix, producing a spectral shift that separates the two bands as well as an amplitude reduction of the fundamental band, and an amplitude enhancement of the overtone or combination band.

The experimental spectra with polarization perpendicular to the scattering plane are compared to synthetic spectra obtained as combination of several Gaussian curves. The number of Gaussian curves is determined by a peak-finder algorithm applied to high temperature spectra so that more peaks can be identified. The peak locations are kept constant for each species as the temperature varies. The peak finder algorithm smooths the first derivative of the signal, and then takes only the zero crossings whose slope exceed a threshold, and with original signal above an amplitude threshold. Details on the peak finder algorithm are described in Ref. [23]. The amplitude and the width of the Gaussian curves are determined by minimizing the difference between the experimental and the computed spectra at each temperature. If the fit is unsatisfactory, the number of peaks is increased, by decreasing the thresholds in the peak-finder algorithm. The spectra are divided in several regions (typically 6 or 7), each containing peaks of approximately the same magnitude. This provides better peak recognition and improves the quality of the fit across the entire spectrum. The amplitude dependence on the temperature is approximated by a third order polynomial, and the width by a linear fit. Peak positions and the coefficients of the polynomials for the amplitude and the width are reported in the supplemental materials. We chose Gaussian curves over Lorentzian as basis functions for the synthetic spectra because they provide a better fit to the experimental data. In combustion applications, the synthetic spectra provided here can be utilized in a manner similar to the spectra derived by Ramses quantum mechanical calculations [9] and, after convolution with the appropriate instrument function at the respective instrument dispersion, can be used to generate the temperature, pixel, and spatial location dependent matrix elements for the hybrid Raman data processing approach.

\subsection{Methane}

Methane is a spherical top molecule, with four distinct normal vibrational modes that are Raman active. The $v_{1}$ mode is a symmetric CH-stretch mode centered at $2917 \mathrm{~cm}^{-1}$, the $v_{3}$ is a triply degenerate $\mathrm{CH}$-stretch mode centered at $3019 \mathrm{~cm}^{-1}$, the $v_{2}$ is a doubly degenerate deformation (bending) mode centered at $1534 \mathrm{~cm}^{-1}$, and the $v_{4}$ is a triply degenerate deformation mode centered at $1306 \mathrm{~cm}^{-1}$. The frequencies of the vibrational modes satisfy the relation $v_{1} \approx v_{3} \approx$ 
$2 v_{2} \approx 2 v_{4}$. Because of this relation between the vibrational frequencies, the Raman spectrum is characterized by polyads, with strong interaction between different vibrational modes. Extensive studies of the methane spectrum are available in the literature.[24-27] More recent work from Jourdanneau et al. [17] includes experimental investigation at low pressure (1 amagat), for temperatures ranging from $300 \mathrm{~K}$ to $900 \mathrm{~K}$, and a quantum mechanical model is proposed with parameters obtained by best fit to the experimental spectra.

The first polyad is a dyad and includes the $v_{2}$ and $v_{4}$ modes. The top portion of Fig. 4 shows the Raman spectra of $\mathrm{CH}_{4}$ in the dyad range $1100 \mathrm{~cm}^{-1}$ to $1800 \mathrm{~cm}^{-1}$ obtained with no polarizer in the path. This is an important region for combustion diagnostics because it overlaps the $\mathrm{O}_{2}$ and $\mathrm{CO}_{2}$ spectra. The integrated $\mathrm{CH}_{4}$ Raman signal (per molecule) in this region is $\sim 47 \%$ of the $\mathrm{O}_{2}$ signal, therefore it constitutes a non-negligible crosstalk contribution. For a $\mathrm{CH}_{4}$-air flame with equivalence ratio of 0.7 , the crosstalk contribution of $\mathrm{CH}_{4}$ onto $\mathrm{O}_{2}$ is $\sim 16 \%$ of the $\mathrm{O}_{2}$ signal in the reactants and decreases at higher temperatures. The black, continuous, vertical lines identify the $\mathrm{CO}_{2}\left(1133-1489 \mathrm{~cm}^{-1}\right)$ and $\mathrm{O}_{2}\left(1489-1662 \mathrm{~cm}^{-1}\right)$ Raman channels at the bowing center; the dotted lines indicates those same channels at the edge of the probe line, shifted spectrally by the bowing effect. Raman spectra are shown in the 300-860 K range. The spectra are dominated by the $v_{2}$ doubly degenerate bending mode at $1534 \mathrm{~cm}^{-1}$. The $v_{4}$ bending mode centered at $1306 \mathrm{~cm}^{-}$ 1 is much weaker, as already observed by Jordanneau et al.,[17] and it is not clearly distinguishable in the experimental spectra. The bottom of Fig. 4 shows the component of the methane Raman signal perpendicularly polarized. The top and bottom plots of the figure differ only by the reduced intensity of the polarized spectra, roughly half of the value measured with no polarizer. This suggests that the dyad scattering is completely anisotropic and not polarized as already observed in [17]. A polarization separation approach such as the one illustrated in [16] would greatly reduce the dyadic contribution of the $\mathrm{CH}_{4}$ spectrum improving the accuracy of $\mathrm{CO}_{2}$ and $\mathrm{O}_{2}$ concentration measurements. The spectral resolution is sufficient to resolve the $\mathrm{S}$ and $\mathrm{O}$ branch transitions $(\Delta \mathrm{J}= \pm 2$ respectively), and the $\mathrm{P}$ and $\mathrm{R}$ branch transitions $(\Delta \mathrm{J}= \pm 1$ respectively), but it is inadequate to resolve the ro-vibrational transitions in the Q-branch. Figure 3 shows a weak temperature dependence of the integrated signal in the dyadic region, first decreasing by $5 \%$ at $600 \mathrm{~K}$ and then increasing back to its original value at $\sim 900 \mathrm{~K}$. Different temperature dependences are observed if the integration region is split to match the $\mathrm{CO}_{2}$ and $\mathrm{O}_{2}$ channels. The integrated spectrum over the $\mathrm{CO}_{2}$ channel increases by $19 \%$ from $300 \mathrm{~K}$ to $860 \mathrm{~K}$, whereas the integrated signal in the $\mathrm{O}_{2}$ channel decreases down to $77 \%$ of its original value. The different temperature dependence of the two channels can be understood by noting that at high temperatures the Stokes transitions from vibrationally excited states (hot-bands) become more significant and the ground vibrational state population diminishes. Because of anharmonic vibrational effects these hot-bands are shifted to lower frequencies relative to transitions from the vibrational ground state. Thus these transitions are shifted preferentially away from the $\mathrm{O}_{2}$ channel and into the $\mathrm{CO}_{2}$ channel. Figure 4 clearly shows the appearance of a frequencydownshifted "shoulder" at high temperatures arising from the Q-branch from the first excited state. The change in the Raman crosstalk between the center and the edge of the probe volume $(+/-3 \mathrm{~mm}$ from optical axis), caused by the "bowing effect" is $-5 \%$ at $300 \mathrm{~K}$ and $-10 \%$ at $860 \mathrm{~K}$ for $\mathrm{O}_{2}$ and $-15 \%$ at $300 \mathrm{~K}$ and $-5 \%$ at $860 \mathrm{~K}$ for $\mathrm{CO}_{2}$. As discussed in the introduction, the error on the $\mathrm{O}_{2}$ and $\mathrm{CO}_{2}$ species concentration measurements when neglecting the change in the methane crosstalk due to the $\mathrm{CH}_{2}$ bending caused by the bowing effect is significantly smaller because of the lower Raman signal intensity in this region, compared to the $\mathrm{O}_{2}$ and $\mathrm{CO}_{2}$ Raman signal. As an example the error in $\mathrm{O}_{2}$ concentration in the reactants of a $\mathrm{CH}_{4}$-air flame with 
equivalence ratio of 0.7 is $\sim 0.8 \%$ of the measured $\mathrm{O}_{2}$ concentration. The dotted spectra in the lower portion of Fig. 4 are the synthetic spectra, obtained as sum of 119 Gaussian curves. The synthetic spectra are optimized so that the integrated signals associated with the $\mathrm{O}$ and $\mathrm{P}$ branch (1150-1512 $\left.\mathrm{cm}^{-1}\right)$, Q branch $\left(1512-1560 \mathrm{~cm}^{-1}\right), \mathrm{S}$ and $\mathrm{R}$ branch $\left(1560-1780 \mathrm{~cm}^{-1}\right)$, match the experimental values.

Figure 5 and Fig. 6 show the methane spectra in the pentad region between 2600 and 3300 $\mathrm{cm}^{-1}$. This is the region typically assigned to the hydrocarbons in Raman measurements when the matrix inversion or the hybrid approach is used. Spectra are dominated by the strong $v_{1}$ totally symmetric stretch mode at $2917 \mathrm{~cm}^{-1}$. This mode has $\mathrm{a}_{1}$ symmetry species, and the associated selection rule is $\Delta \mathrm{J}=0$, allowing only Q-branch transitions. Figure 5 shows a close-up of the perpendicular component of the methane Raman spectra in the $2890-2925 \mathrm{~cm}^{-1}$ region, corresponding to the Q-branch of the $v_{1} \mathrm{CH}$-stretch mode. Spectral resolution is not sufficient to resolve the ro-vibrational structure. As the temperature increases, the amplitude of the $2917 \mathrm{~cm}^{-1}$ peak decreases, but additional peaks appear at lower frequencies (Q-branches of the hot bands) due to the increase in the population of levels with higher energies. The integrated signal associated with this region $\left(2890-2925 \mathrm{~cm}^{-1}\right)$ stays within $1 \%$ of the ambient temperature value. Figure 6 compares the perpendicular (upper plot) and parallel (lower plot) components of the Raman spectra. The strongly isotropic $v_{1}$ mode [17] is almost completely perpendicularly polarized, and its intensity is significantly reduced in the parallel component. The dominant features in the parallel component of the spectra are associated with the ro-vibrational structure of the triply degenerate, $v_{3}$ stretching mode $\left(\mathrm{f}_{2}\right.$ symmetry species, with selection rules $\Delta \mathrm{J}=0, \pm 1$, \pm 2 ). Hot bands for this mode are too weak to be observed in this temperature range. The expanded vertical scale of Fig. 6, allows one to discern additional isotropic (polarized) features, associated with the first overtone of the deformation modes $\left(2 v_{4}\right.$ with a peak at $2586 \mathrm{~cm}^{-1}$ and $2 v_{2}$ with a peak at $3073 \mathrm{~cm}^{-1}$ ) and the $v_{2}+v_{4}$ combination mode at $2835 \mathrm{~cm}^{-1}$. These modes are enhanced by Fermi resonance with the $v_{1}$ stretching mode. Additional Raman signal is expected in the octad region near $4300 \mathrm{~cm}^{-1}$. It is produced only by overtones and combination modes, but its contribution is too weak and can be neglected for combustion applications. The synthetic spectra for the perpendicular component of the Raman spectra are obtained as sum of 166 Gaussian curves. The synthetic spectra are intended to be used with the hybrid approach, therefore small discrepancies in the spectral features are negligible as long as the integrated signal is conserved. The integrated Raman signal in the $\mathrm{CH}$-stretch region $\left(2548-3434 \mathrm{~cm}^{-1}\right)$ depends only weakly on temperature (increase up to $3 \%$ ) as shown in Fig. 3. When multiple hydrocarbons are measured simultaneously, the $\mathrm{CH}$-stretch region is divided in several hardware-binned regions to define separate channels for all the hydrocarbons present. Each region has a different temperature dependence that can be extracted by convolving the synthetic spectra with the appropriate instrument function and then integrating over the spectral region associated with each channel.

\subsection{Ethylene}

The ethylene molecule $\left(\mathrm{H}_{2} \mathrm{C}=\mathrm{CH}_{2}\right)$ is a prolate asymmetric rotor, with twelve nondegenerate normal vibrational modes, 6 of which are Raman active. Extensive literature is available on ambient temperature Raman spectra of ethylene. Foster et al. presented detailed studies on the $v_{1}$, $v_{3}$ and $v_{5}$ Raman vibrational bands [28]; Knippers et al. [29] investigated some of the overtone Raman Q branches. More recently, Bermejo et al. [30] investigated the $v_{2}, v_{3}$, and $2 v_{10}$ Raman bands at very high resolution $\left(0.003 \mathrm{~cm}^{-1}\right)$. Oomens et al. [31] investigated the hot bands in the 
$2970-3015 \mathrm{~cm}^{-1}$ region at low rotational temperatures by pumping the molecules to excited states using an IR-IR double resonance technique. For combustion application a mixture of methane and ethylene is a commonly used JP7 surrogate. [14] CARS measurements of ethylene in flames have been demonstrated by Beyrau et al. [32]. Raman measurements of ethylene in flames are severely limited by the low photo-fragmentation threshold and by $\mathrm{C}_{2}$ fluorescence interference effects. We recently developed a polarization-separation Raman/Rayleigh instrument [16] to remove the effect of $\mathrm{C}_{2}$ fluorescence interference from the Raman data on a single-shot basis. [16] As shown in Fig. 1 the Raman spectra of methane and ethylene are sufficiently separated also with the low-dispersion grating, and the hybrid approach can be applied to data from the polarization separation Raman instrument, if ethylene and methane spectra are present. Despite the large interest in combustion applications, studies of the evolution of the ethylene Raman spectra at higher temperature are scarce. Quantum mechanical models for the ethylene Raman spectrum are not available in the open literature.

Figure 7 shows the ethylene spectra in the $1100-1780 \mathrm{~cm}^{-1}$ range for temperatures between 300 and $860 \mathrm{~K}$. The strong spectral peak at $1343 \mathrm{~cm}^{-1}$ is associated with the $v_{3}$ normal vibrational mode $\left(\mathrm{CH}_{2}\right.$ bending). The rotational structure associated with this $\mathrm{Q}$-branch is very compressed and it is unresolved in our data. Weak and broad $\left(\sim 400 \mathrm{~cm}^{-1}\right)$ wings, presumably unresolved O, P R and S branch lines, accompany the Q-branch, but are not visible at the vertical scale used in Fig. 7. The $v_{3}$ has $\mathrm{a}_{\mathrm{g}}$ symmetry therefore is mostly isotropic and strongly polarized. The component of the spectra shows only a small reduction in the peak signal (13\%) with respect to the spectra taken with no polarizer (not shown here, but available in tabular form), confirming that this vibrational structure is prevalently isotropic and strongly polarized. The unresolved $\mathrm{O}$, $\mathrm{P}, \mathrm{R}$ and $\mathrm{S}$ branch lines that constitute the wings are anisotropic and, as expected, their amplitude is roughly halved in the polarized spectra. At higher temperatures the $v_{3}$ band becomes broader and skewed toward lower frequencies, as a consequence of the appearance of hot bands that are unresolved at this resolution. As the temperature increases, the $v_{3}$ signal integrated over the $\mathrm{CO}_{2}$ channel $\left(1133-1489 \mathrm{~cm}^{-1}\right)$, normalized by the density, increases by up to $23 \%$. The integrated Raman signal associated with the $v_{3}$ mode at $300 \mathrm{~K}$ is $\sim 3.43$ time larger than the $\mathrm{N}_{2}$ signal, and falls completely in the $\mathrm{CO}_{2}$ channel. Since the spectral features are well centered in the $\mathrm{CO}_{2}$ channel, the crosstalk correction is not sensitive to beam steering or to the bowing effect. The region corresponding to the $\mathrm{O}_{2}$ channel $\left(1470-1660 \mathrm{~cm}^{-1}\right)$ is dominated by the $v_{2}$ vibrational mode of ethylene, a $\mathrm{C}_{2}$ stretching mode centered at $1624 \mathrm{~cm}^{-1}$, and the second overtone of the $v_{10}$ vibrational mode $\left(\mathrm{CH}_{2}\right.$-bend), expected around $1652 \mathrm{~cm}^{-1}$, which is enhanced by Fermi resonance and produces the weak broad structures on the blue side of the $v_{2} \mathrm{Q}$-branch. The spectra associated with the $v_{2}$ mode fall completely within the $\mathrm{O}_{2}$ channel, and the integrated "crosstalk" contribution is not sensitive to beam steering and to the bowing effect. At $300 \mathrm{~K}$ the integrated signal in this region is $\sim 1.7$ time the signal in $\mathrm{N}_{2}$, and therefore constitutes a major crosstalk contribution even at low concentrations. Similar to the $v_{3}$ Q-branch, the spectrum is strongly polarized with only a $17 \%$ reduction in the peak intensity in the perpendicular component. The spectra show strong temperature dependence, with the peak at $1624 \mathrm{~cm}^{-1}$ down to $14 \%$ of its original amplitude when the temperature reaches $860 \mathrm{~K}$. In turn lower frequencies hot bands become stronger, and the integrated signal over the $\mathrm{O}_{2}$ channel $\left(1470-1660 \mathrm{~cm}^{-1}\right)$ after a small $3 \%$ drop at $400 \mathrm{~K}$ increases at $860 \mathrm{~K}$ by $\sim 6 \%$ relative to room temperature. Synthetic spectra were obtained for the perpendicular polarization and plotted as dashed curves in Fig. 7. The $v_{3}$ mode is represented by six Gaussian curves, where the spectral features in the $\mathrm{O}_{2}$ channel are approximated by combinations of 37 Gaussian curves. Small discrepancies can be observed 
on the low frequency side of the $v_{3}$ mode; the integrated signal obtained from the synthetic spectra is within $0.2 \%$ of the measured value.

Figure 8 shows the perpendicular component of the $v_{1}$ band $\left(\mathrm{CH}\right.$ stretch, $\mathrm{a}_{\mathrm{g}}$ symmetry species) of the ethylene Raman spectra in the $2980-3050 \mathrm{~cm}^{-1}$ region. The band is characterized by the strong Q-branch transition peaking at $3020 \mathrm{~cm}^{-1}$. As the temperature increases the peak amplitude drops quickly as hot bands become stronger, but the total integrated signal is roughly constant. Hot bands appear as the temperature increases, but the spectral resolution is not sufficient to resolve them, and only a broadening of the spectra is observed. The $v_{1} \mathrm{Q}-\mathrm{branch}$ is highly isotropic, and removing the polarizer only increases its intensity slightly.

Figure 9 shows the perpendicular component of the Raman spectra of ethylene in the 2600$3350 \mathrm{~cm}^{-1}$ region, with a significantly expanded vertical scale, to allow visualization of minor contribution to these spectra. The broad spectral structures centered at $3102 \mathrm{~cm}^{-1}$ are associated with the $v_{5} \mathrm{CH}_{2}$ stretching mode. The spectral features centered at $2878 \mathrm{~cm}^{-1}$ are attributed to the second overtone of the $v_{12} \mathrm{CH}_{2}$ in plane bending mode. The peak at $3240 \mathrm{~cm}^{-1}$ is attributed to the second overtone of the $v_{2} \mathrm{C}_{2}$ stretching mode, and the $3274 \mathrm{~cm}^{-1}$ peak is attributed to the $v_{2}+2 v_{10}$ mode $\left(\mathrm{C}_{2}\right.$ stretch and second overtone of the $\mathrm{CH}_{2}$ rocking mode). Comparison of spectra obtained with and without the polarizer shows that all those vibrational modes are highly isotropic and perpendicularly polarized except for the $v_{5}$ asymmetric stretch mode that is completely depolarized.

Significant changes in the spectra are observed with changes in the temperature. The $v_{1}$ mode behaves similarly to the previously described $v_{2}$ mode; the peak value decreases rapidly (to $17 \%$ of the room temperature value for the $3020 \mathrm{~cm}^{-1}$ peak), but lower frequency hot bands become stronger, producing a skewed broadening of the lines at this low resolution. The integrated Raman signal decreases by $2 \%$ from $300 \mathrm{~K}$ to $400 \mathrm{~K}$ and then increases monotonically with temperature, up to $4 \%$ at $860 \mathrm{~K}$. The amplitudes of the overtone modes quickly decrease with temperature and at $860 \mathrm{~K}$ cannot be distinguished from the broad wings of the $v_{1}$ mode. The synthetic spectra in the $\mathrm{CH}$-stretch region are a combination of 137 Gaussian curves. Discrepancies are observed mostly for the $v_{1}$, making the synthetic spectra inadequate for a spectral fitting approach. The integrated area of the synthetic spectra differ by less than $0.15 \%$ from the measured value, and the discrepancies in the spectral features are not significant for the evaluation of the matrix elements in the hybrid approach.

\subsection{Ethane}

The ethane molecule has twelve fundamental vibration modes, but only six are Raman active. There is a discrepancy in the numbering and the symmetry designation of modes $v_{7}$ to $v_{12}$ reported by van Helwoort [33] and by Shimanouchi [22]. As for the remaining of the paper we follow Shimanouchi [22] numbering that is also consistent with NIST tables. The 3 modes $\left(v_{1}, v_{2}\right.$, $v_{3}$ ) belonging to the totally symmetric $\mathrm{a}_{\mathrm{g}}$ symmetry species are vibrational-rotational bands that satisfy the selection rule $\Delta \mathrm{K}=0$ and $\Delta \mathrm{J}= \pm 1$, where the three degenerate modes $\left(v_{7}, v_{8}, v_{9}\right)$ have Raman bands that satisfy $\Delta \mathrm{K}= \pm 1, \pm 2$ and $\Delta \mathrm{J}=0, \pm 1, \pm 2$. [33] Extensive studies of the Raman spectrum of ethane at room temperature are available in the literature. [33-37] Ethane is present in significant amounts in natural gas, and therefore it is of interest for combustion applications. It is also one of the major intermediates in DME combustion, which is of current research interest. Other than the low-resolution study by Ekoto et al.,[18] there are no experimental databases that provide the Raman spectra of ethane as a function of the temperature. 
Figure 10 shows the ethane spectrum in the $880-1100 \mathrm{~cm}^{-1}$ region. In the hybrid approach this region is assigned to the signal from the $\mathrm{C}_{2}$ fluorescence interference, and used as a basis for computing the corrections needed to remove this interference in most Raman channels. Thus, failure to account for the ethane contribution in this region will propagate to all the other concentration measurements. The ethane spectrum in this region is dominated by the $\mathrm{C}_{2}$ stretch mode centered at $995.3 \mathrm{~cm}^{-1}$. The Q-branch transitions are strongly polarized, where the weak O and $\mathrm{S}$ branch are not polarized. The spectra in this region are strongly temperature dependent. The spectral resolution available is not sufficient to resolve the hot band, but the envelope changes dramatically, producing large shifts in the peak location, and increasing the width and the skewness of the spectral features. The integrated signal increases by $67 \%$ when the temperature rises from $300 \mathrm{~K}$ to $860 \mathrm{~K}$. The synthetic spectra for the perpendicular component are obtained as a combination of 59 Gaussian curves. No major discrepancies are visible in the figure and the integrated signals from the synthetic spectra are within $1 \%$ of the measured values.

Figure 11 shows the ethane spectrum in the $1100-1800 \mathrm{~cm}^{-1}$ region corresponding to the $\mathrm{CO}_{2}$ and $\mathrm{O}_{2}$ channels. Spectra obtained with no polarizer (top) and with the polarizer oriented to transmit the perpendicular polarization (bottom) are shown. The major feature in this region is attributed to the $v_{8} \mathrm{CH}_{3}$ deformation mode centered at $1469 \mathrm{~cm}^{-1}$. The Raman spectra show an unresolved envelope that extends up to $250 \mathrm{~cm}^{-1}$ from the band origin, overlapped with well resolved peaks, regularly spaced about $6-7 \mathrm{~cm}^{-1}$ apart. This regular structure is associated directly with the $\mathrm{P}, \mathrm{O}, \mathrm{Q}, \mathrm{R}$ and $\mathrm{S}$ branches of the $v_{8}$ band, and it is described in detail in [33]. The non-resolved envelope is attributed to complex interactions of the $v_{8}$ band with several combination bands and overtones such as $3 v_{4}+v_{12}, 2 v_{4}+v_{9}$, and $v_{4}+v_{12}$. Very weak, depolarized features associated with the $v_{9}$ normal mode $\left(\mathrm{CH}_{3}\right.$ rocking) are centered at $1195 \mathrm{~cm}^{-1}$. The narrow peaks at $1343 \mathrm{~cm}^{-1}$ and $1642 \mathrm{~cm}^{-1}$ do not belong to ethane but are associated with the $v_{2}$ and $v_{3}$ modes of ethylene. The ethane gas was provided by Matheson cylinder with a $\mathrm{CP}$ grade that guarantees $>99 \%$ ethane, and from the peak height we estimate a mole fraction of $\sim 0.25 \%$ of ethylene. Comparison between the spectra obtained with and without the polarizer show that the $v_{8}$ is not polarized, and its intensity is reduced by roughly half when adding a polarizer. The polarized $2 v_{12}$ transition at $\sim 1646 \mathrm{~cm}^{-1}$ observed in Ref.[33] is noticeable in the perpendicular component, but it is very weak. The ethane signal in the $\mathrm{CO}_{2}$ and $\mathrm{O}_{2}$ region is roughly 0.75 of the $\mathrm{N}_{2}$ signal when collecting only the perpendicular polarization, and approximately twice that value if no polarizer is used. The Raman signal increases with temperature, reaching a $10 \%$ increment at $860 \mathrm{~K}$. The signal in the $\mathrm{CO}_{2}$ channel decreases with temperature reaching a minimum of $93 \%$ of the ambient temperature value at $500 \mathrm{~K}$ and is then slowly increasing. The signal in the $\mathrm{O}_{2}$ channel increases monotonically with temperature reaching a 5\% increment at $860 \mathrm{~K}$. The ethane crosstalk in the $\mathrm{O}_{2}$ and $\mathrm{CO}_{2}$ channels is strongly dependent on the spatial location along the probe volume due to the bowing effect, with variations of up to $-26 \%$ and $+20 \%$ between the center of the bowing and the edges for $\mathrm{O}_{2}$ and $\mathrm{CO}_{2}$, respectively. The error is reduced to $-9 \%$ and $+10 \%$ at $860 \mathrm{~K}$. The synthetic spectra are obtained as combinations of 96 Gaussian curves. Discrepancies are visible between the synthetic and the experimental spectra, most likely caused by an insufficient number of peaks, in particular near the $v_{9}$ and the $2 v_{12}$ modes. The integrated signal over the combined $\mathrm{CO}_{2}-\mathrm{O}_{2}$ channels from the synthetic spectra is within $2 \%$ of the experimental value; the integrated signal over the $\mathrm{CO}_{2}$ is up to $2.7 \%$ smaller than the experimental value, and the integrated signal over the $\mathrm{O}_{2}$ channel is up to $3 \%$ larger. Nevertheless, the synthetic spectra allow accounting for beam steering and for the bowing effect, greatly reducing the error in the ethane crosstalk terms. 
Figure 12 shows the ethane spectrum in the $2700-3100 \mathrm{~cm}^{-1}$. Two Raman-active fundamental vibrational modes are present in this region: the strong $v_{1}$ symmetric $\mathrm{CH}_{3}$ stretch mode centered at $2953.7 \mathrm{~cm}^{-1}$ and the weaker $v_{7}$ asymmetric $\mathrm{CH}$-stretch mode centered at $2968.7 \mathrm{~cm}^{-1}$. The other major features in this region are the peaks at 2894 and $2899 \mathrm{~cm}^{-1}$, corresponding to a very strong Fermi resonance of the overtones of the in phase $\left(v_{8}\right)$ and out of phase $\left(v_{11}\right)$ asymmetric $\mathrm{CH}_{3}$ deformation with the symmetric stretching mode $v_{1}$. [33] As a result of the Fermi resonance the overtones amplitude is greatly increased and their frequencies blue shifted by 45 and $42 \mathrm{~cm}^{-1}$ respectively. Hot bands associated with the $v_{1}$ and $2 v_{8}$ Q-branch transitions are responsible for the skewness toward the red. Weaker but well defined spectral features appear at $2744 \mathrm{~cm}^{-1}$ attributed to the $v_{6}$ overtone, and at $2779 \mathrm{~cm}^{-1}$, attributed to the $v_{2}$ overtone (with its blue shifted $2 v_{2+} v_{4}$ hot band), enhanced by anaharmonic resonance with the fundamental $v_{1}$ band. [33] Comparison of the perpendicular and parallel components of the Raman spectra shows that the $v_{1}, 2 v_{8} 2 v_{6}$ and $2 v_{2}, 2 v_{2+} v_{4}$ modes are primarily isotropic and strongly polarized. Because of the large collection angle, the peaks at $2899 \mathrm{~cm}^{-1}$ and $2954 \mathrm{~cm}^{-1}$ are still visible in the parallel component. A large broadband, unpolarized, envelope, similar to the one produced by the $v_{8}$ mode, covers the $2800-3100 \mathrm{~cm}^{-1}$ range in the parallel polarization plot, and it is attributed to the $v_{7}$ mode ( $\mathrm{e}_{\mathrm{g}}$ symmetry) centered at $2969 \mathrm{~cm}^{-1}$, with additional contributions from the anisotropic component of the $v_{1}$ mode.

The ethane spectrum in the $2700-3100 \mathrm{~cm}^{-1}$ region shows strong temperature dependence. The peak amplitude decreases, hot bands appear toward lower frequencies and become dominant, causing a shift of the maximum amplitude location, a broadening, and an increased skewness of the $v_{1}, 2 v_{8}$ and $2 v_{11}$ modes. The $v_{7}$ depolarized mode shows a strong rotational dependence with the temperature, with a broadening of the envelope, with amplitude increasing on the red side of the center band and decreasing on the blue side. The total integrated signal is only weakly dependent on the temperature, remaining within $3 \%$ of the ambient temperature value throughout the entire range considered. The synthetic spectra are obtained as combinations of 67 Gaussian curves. We observe discrepancies between the experimental and synthetic spectra near the $v_{8}$ and $v_{11}$ overtones, but good agreement everywhere else. The difference between the integrated signals over the entire $\mathrm{CH}$-stretch region is within $2 \%$.

\subsection{Dimethyl ether}

Dimethyl ether (DME) is one of the simplest and most abundant ethers, and there is extensive literature on the ambient temperature spectra. [38-42] Recently, there has been an increased interest in the combustion community because it is a renewable biofuel with good auto-ignition properties, and it is a promising alternative diesel fuel. DME has two of the moment of inertia very close to each other, therefore can be approximated to a symmetric top molecule. [40] The molecule has 21 normal vibrational modes, 13 of which are Raman active. There is a discrepancy in the numbering and the symmetry designation of modes $v_{15}$ to $v_{21}$ reported by Coudert et al. [41] and by Shimanouchi [22]. To be consistent with the remaining of the paper, and with what reported in the NIST tables, [43] we use Shimanouchi [22] numbering. Experimental spectra shown here are limited to $690 \mathrm{~K}$ because at higher temperatures $\mathrm{CH}_{4}$ and $\mathrm{CH}_{2} \mathrm{O}$ are formed by pyrolysis due to contact with the heating element which is at a higher temperature than the probe volume.

Figure 13 shows the perpendicular component of the DME spectra in the $900-1200 \mathrm{~cm}^{-1}$ region. The integrated signal in this region is $\sim 3.5$ times the $\mathrm{N}_{2}$ signal. The strongest feature in this region is associated with the symmetric $\mathrm{CO}$ stretch mode $\left(\mathrm{v}_{6}, \mathrm{a}_{1}\right.$ symmetry species) centered 
at $932 \mathrm{~cm}^{-1}$. This mode shows a strongly polarized Q-branch and a poorly resolved, broad baseline that is not polarized attributed to the $\mathrm{O}, \mathrm{P}, \mathrm{R}$ and $\mathrm{S}$ branches (spectra with no polarizer are not shown here, but available in tabular form). The broad, featureless signal from 1000 to $1200 \mathrm{~cm}^{-1}$ is a combination of the $\mathrm{v}_{10} \mathrm{CH}_{3}$ rocking mode, centered at $1150 \mathrm{~cm}^{-1}$, and the $\mathrm{v}_{17}$ asymmetric $\mathrm{CO}$ stretch mode, centered at $1104 \mathrm{~cm}^{-1}$. The amplitudes of these modes are approximately halved when adding a polarizer suggesting that they are anisotropic. The $\mathrm{v}_{6}$ mode is strongly temperature dependent; lower frequency transitions become dominant at lower temperature (hot bands), causing a shift and a broadening of the unresolved spectrum. The underlying depolarized modes instead become weaker when increasing the temperature. The integrated signal in the region increases rapidly with temperature, reaching a $15 \%$ increment at $690 \mathrm{~K}$. The synthetic spectra are generated by 21 Gaussians, providing an integrated signal that is within $2.5 \%$ of the experimental value.

Figure 14 shows the perpendicular component of the Raman spectra collected in the 1215$1775 \mathrm{~cm}^{-1}$ region. The expected [22] $\mathrm{CH}_{3}$ rocking mode at $1227 \mathrm{~cm}^{-1}$ is too weak, and is not visible in the recorded spectra. The $1300-1600 \mathrm{~cm}^{-1}$ region is characterized by a complex spectrum generated by the $v_{3}$ symmetric $\mathrm{CH}_{3}$ bend mode, two symmetric $\mathrm{CH}_{2}$ bend modes $\left(v_{4}\right.$, $\left.v_{19}\right)$ and three asymmetric $\left(v_{9}, v_{14}, v_{15}\right) \mathrm{CH}_{2}$ bend modes all having frequencies centered around $1450 \mathrm{~cm}^{-1}$.[44] Removing the polarizer the spectral shape is unaltered, but the amplitude roughly doubled indicating that the modes are not polarized and anisotropic. The weak, polarized peak at $1244 \mathrm{~cm}^{-1}$ was attributed to the symmetric deformation mode $v_{5}$ by Taylor et al. [38] The spectrum in the $1300-1600 \mathrm{~cm}^{-1}$ region shows a strong temperature dependence; at higher temperature the spectrum becomes wider, the peak amplitudes smaller, the center region becomes featureless and weaker, and the wings stronger. This region falls between the $\mathrm{CO}_{2}$ and $\mathrm{O}_{2}$ Raman channels. The signals in the $\mathrm{O}_{2}$ and $\mathrm{CO}_{2}$ channels are $\sim 0.53$ and 1.7 times the $\mathrm{N}_{2}$ signal, respectively. The signal in the $\mathrm{CO}_{2}$ channel decreases with temperature by $\sim 6 \%$ at $690 \mathrm{~K}$, while the signal in the $\mathrm{O}_{2}$ channel increases monotonically up to 1.6 times the ambient temperature value. Neglecting the bowing effect, the DME crosstalk at the edge of the probe volume is overestimated by $11 \%$ for $\mathrm{CO}_{2}$, and underestimated by $54 \%$ for $\mathrm{O}_{2}$. The synthetic spectra are obtained as combinations of 8 Gaussian curves and do not adequately represent the detailed spectral features. The integrated area in the $\mathrm{CO}_{2}$ channel is within $2 \%$ of the experimental values, but errors up to $6 \%$ are found in the $\mathrm{O}_{2}$ channel. The error could be reduced if a higher number of peaks were used for the synthetics spectra.

The CH-stretch (2700-3100 $\mathrm{cm}^{-1}$ ) region of the Raman spectra of DME, shown in Fig. 15 is dominated by the $v_{1}$ symmetric $\mathrm{CH}$ stretch mode at $2993 \mathrm{~cm}^{-1}$, the $v_{12}$ asymmetric $\mathrm{CH}$ stretch mode at $2991 \mathrm{~cm}^{-1}$, two symmetric $\mathrm{CH}_{2}$ stretch modes $\left(v_{2}\right.$ at $2822 \mathrm{~cm}^{-1}$ and $v_{13}$ at $2820 \mathrm{~cm}^{-1}$ ) and an asymmetric $\mathrm{CH}_{2}$ stretch modes $\left(v_{8}\right.$ at $\left.2893 \mathrm{~cm}^{-1}\right)$ [22]. The frequencies of the peaks in the experimental spectra are shifted by the strong Fermi resonance with combination and overtone modes. The total Raman signal in this region is $\sim 21$ times the $\mathrm{N}_{2}$ signal. The experimental data show the strongest peaks at $2819 \mathrm{~cm}^{-1}$ associated with the $v_{2}$ and the $v_{13}$ symmetric $\mathrm{CH}_{2}$ stretch modes, and at $\sim 2998 \mathrm{~cm}^{-1}$ associated with the $v_{1}$ symmetric $\mathrm{CH}$ stretch $\left(2993 \mathrm{~cm}^{-1}\right)$ and the $v_{12}$ asymmetric $\mathrm{CH}$ stretch $\left(2991 \mathrm{~cm}^{-1}\right)$ modes. Additional experimental peaks appear at 2870, 2889, $2899,2921,2935$, and $2960 \mathrm{~cm}^{-1}$. The spectra are complicated by the numerous overtones and combinations of the $\mathrm{CH}_{3}$ deformation modes that land in this region. These numerous modes are also anharmonically shifted and amplified by Fermi resonance, because of their spectral proximity to the $v_{1}$ and $v_{2}$ modes. Comparison of the data collected with no polarizer and with the vertical and horizontal polarizers shows that the spectrum is strongly polarized, but also reveals a 
broad, much weaker, unpolarized component, centered around $2974 \mathrm{~cm}^{-1}$ and $\sim 400 \mathrm{~cm}^{-1}$ broad. The DME spectrum in the $\mathrm{CH}$-stretch region shows very strong temperature dependence. The amplitude of peaks at 2819, 2870, and $2998 \mathrm{~cm}^{-1}$ decreases at higher temperature, while their width increases. The peak at $2960 \mathrm{~cm}^{-1}$ quickly disappears with increasing temperature, but new features appear around $2940 \mathrm{~cm}^{-1}$, merging with the peak at $2936 \mathrm{~cm}^{-1}$, whose amplitude and width increase at higher temperature. The parallel component of the spectrum shows only weak temperature dependence, characterized by a broadening of the unresolved structure. The integrated signal from the perpendicular component of the Raman signal increases with temperature by $4 \%$ at $690 \mathrm{~K}$, while the parallel component stays within $1 \%$ of the ambient temperature value. The synthetic spectra are obtained as combinations of 61 Gaussians, and comparison with the experimental spectra show good agreement. The integrated signal is within $1 \%$ of the measured value.

\subsection{Formaldehyde}

Figure 16 shows Raman spectra collected in a DME jet heated to $780 \mathrm{~K}$ and $860 \mathrm{~K}$. Locally, near the heater element itself, DME reaches temperatures higher than what is measured by the thermocouple located downstream at the exit, and pyrolysis occurs, producing methane and formaldehyde. DME spectrum at $860 \mathrm{~K}$ shows the $v_{3} \mathrm{CH}_{2}$ scissor mode of formaldehyde centered at $1499.7 \mathrm{~cm}^{-1}$, the weak $v_{2} \mathrm{CO}$ stretch mode at $1742 \mathrm{~cm}^{-1}$, and the strong $v_{1} \mathrm{CH}_{2}$ stretch mode centered at $2782 \mathrm{~cm}^{-1}$. [45] The $v_{4} \mathrm{CH}_{2}$ asymmetric stretch mode lands in a region with a strong DME contribution and it is too weak to detect. The strong $v_{1}$ mode of methane at $2917 \mathrm{~cm}^{-1}$ is also evident at $860 \mathrm{~K}$. Formaldehyde is a molecule of great interest for combustion applications, but experimental studies of its Raman spectra in the gas phase are very scarce and limited only to ambient temperature. [45, 46]

To investigate the behavior of the formaldehyde at lower temperatures we added $\mathrm{N}_{2}$ to the flow through a previously capped port shown in Fig. 2. The cold $\mathrm{N}_{2}$ flow mixed with the heated mixture of DME methane and formaldehyde, after pyrolysis occurred, and temperatures as low as $400 \mathrm{~K}$ were reached. The spectra in Fig. 17 are obtained by subtracting the synthetic spectra for methane and DME at the measured temperature from the experimental spectra. The spectra are normalized so that the total signal associated to formaldehyde is constant with temperature. The concentration of formaldehyde in the sample was not constant, and therefore no information about the dependence of the integrated signal with temperature could be extracted. The spectra show that as the temperature increases hot bands appear, and the intensity of the $2782 \mathrm{~cm}^{-1}$ peak decreases. To our knowledge this is the first time Raman spectra of formaldehyde at temperature up to $860 \mathrm{~K}$ have been reported. The lack of a temperature dependent profile will certainly affect the accuracy of formaldehyde concentration measurements in flames, but comparison with other hydrocarbon Q-branches suggests that the error will be less than $2 \%$ in the $300-900 \mathrm{~K}$ range. Larger discrepancies may be caused by errors in the DME synthetic spectra, in particular in the presence of apparent spectral shifts caused by beam steering or by the bowing effect. Since ambient temperature measurements in pure formaldehyde are not available, we also lack an absolute calibration.

\subsection{Propane}

Propane is a very common fuel, and Raman spectroscopy has been previously applied to propane-air flames. Early studies [47] have identified the contributions of the 27 distinct vibrational modes to the Raman spectra spectrum of propane, but neither quantum mechanical 
models nor experimental studies at higher temperatures are available. Figure 18 shows Raman spectra of propane in the $900-1800 \mathrm{~cm}^{-1}$ region. Because of the low spectral resolution, the rotational structures are unresolved. The integrated signal in this region is $\sim 3.6$ times the $\mathrm{N}_{2}$ signal. Reference [47] indicates that the strongest peak is centered at a Raman shift of $867 \mathrm{~cm}^{-1}$ which is outside the spectral region accessible in these experiments. The broad structure centered at $1054 \mathrm{~cm}^{-1}$ is attributed to the $v_{20} \mathrm{C}_{2}$ stretch mode. The peaks at $1157 \mathrm{~cm}^{-1}$ and $1193 \mathrm{~cm}^{-1}$ are attributed to the $v_{7}$ and to the $v_{25} \mathrm{CH}_{3}$ rocking modes. Weak and broad structure located between 1200 and $1400 \mathrm{~cm}^{-1}$ are associated to the $v_{12} \mathrm{CH}_{2}$ twist mode centered at $1278 \mathrm{~cm}^{-1}$ and the $v_{19}$ $\mathrm{CH}_{2}$ wag mode centered at $1338 \mathrm{~cm}^{-1}$. The region between 1350 and $1600 \mathrm{~cm}^{-1}$ is characterized by the strong $v_{11} \mathrm{CH}_{3}$ deformation mode centered at $1452 \mathrm{~cm}^{-1}$, overlapping a broad structure generated by the interaction between several weaker transitions including $\mathrm{CH}_{3}$ deformation modes $\left(v_{4}, v_{17}\right.$ and $\left.v_{24}\right), \mathrm{CH}_{2}$ scissor mode $v_{5}$, and overtones and combination modes. Comparison between the spectra obtained with and without the polarizer show that all the features are strongly anisotropic except for the $v_{7}$ and $v_{25} \mathrm{CH}_{3}$ rocking modes. The integrated signal in this region shows an increase of $11 \%$ in the $300-690 \mathrm{~K}$ range. The signal integrated over the $\mathrm{C}_{2}$ channel is $\sim 25 \%$ of the signal in this region, and increases by $40 \%$ when increasing the temperature to $690 \mathrm{~K}$. The integrated signal in the $\mathrm{CO}_{2}$ channel accounts for $63 \%$ of the total signal in this region, and decreases by $8 \%$ when the temperature reaches $690 \mathrm{~K}$. The integrated signal in the $\mathrm{O}_{2}$ channel accounts for the remaining $12 \%$ of the total signal, and increases by $60 \%$ in the 300-690 K range. The signal in the $\mathrm{CO}_{2}$ and $\mathrm{O}_{2}$ channel is sensitive to beam steering and to the bowing effect. The propane crosstalk on the $\mathrm{CO}_{2}$ channel is $11 \%$ weaker at the edge of the measurement volume, because a smaller portion of the $v_{11}$ mode falls in the channel. On the other hand the propane crosstalk on $\mathrm{O}_{2}$ is $72 \%$ stronger. The difference in the crosstalk strength between the bowing center and the edges is reduced at higher temperature, being $5.5 \%$ for $\mathrm{CO}_{2}$ and $30 \%$ for $\mathrm{O}_{2}$. The synthetic spectra show good agreement with the experimental data; the computed integrated signal in the $\mathrm{CO}_{2}$ and $\mathrm{O}_{2}$ channels are within 1 and 2.5\%, respectively.

Figure 19 shows the propane spectra in the $2700-3100 \mathrm{~cm}^{-1}$ Raman shift region. The dominant feature is the peak at $2887 \mathrm{~cm}^{-1}$ attributed to the symmetric stretching modes of $\mathrm{CH}_{2}$ $\left(v_{3}\right)$, and $\mathrm{CH}_{3}\left(v_{16}\right)$. At higher frequencies the spectra become more complex because of the interaction, asymmetric stretching modes $v_{10}, v_{15}, v_{22}$, and $v_{1}\left(2967,2968,2973\right.$ and $\left.2977 \mathrm{~cm}^{-1}\right)$ of $\mathrm{CH}_{3}$, with $\mathrm{CH}_{2}$ stretching modes $v_{2}\left(2962 \mathrm{~cm}^{-1}\right.$, symmetric) and $v_{23}\left(2968 \mathrm{~cm}^{-1}\right.$, asymmetric), and several combination and overtone modes. Comparison of the data collected in the two polarization directions shows that distinct, strongly polarized peaks are overlapped with a broad, much weaker, unpolarized component, centered around $2935 \mathrm{~cm}^{-1}$ and $\sim 200 \mathrm{~cm}^{-1}$ broad. The polarized smaller peaks at 2738 and $2771 \mathrm{~cm}^{-1}$ are likely combination modes $\left(v_{5}+v_{12}\right)$ and $\left(v_{18}+v_{6}\right)$, although we cannot be certain of this attribution. The spectra show a strong temperature dependence, with the peak amplitudes becoming lower, but the width larger, resulting in only modest changes in the overall integrated signal (within 3\% for the perpendicular polarization).

\section{Combustion applications: library generation and extrapolation to high temperatures}

The spectra described in the previous section show that for combustion applications it is preferable to collect only the perpendicular component of the Raman spectra. The Raman channel associated to a species is chosen to include the strongest, almost completely polarized transitions of that species, therefore the signal-to-noise ratio is only weakly reduced by collecting only the perpendicular component of the Raman signal. Crosstalks of methane, ethane and DME 
onto the $\mathrm{O}_{2}$ and $\mathrm{CO}_{2}$ channel are attributed to transitions that are not polarized and therefore are roughly halved by rejecting the parallel component of the Raman signal. Similarly, using the high dispersion spectrometer (Fig. 1b) the contributions of each hydrocarbon in the region dominated by another hydrocarbon are often associated to $\mathrm{O}, \mathrm{P}, \mathrm{S}$, and R transitions that are not polarized. For this reason synthetic spectra have been generated only for the perpendicular polarization, but the same approach can be easily extended to the spectra with no polarizer and to the parallel component. The synthetic spectra provided in this work can be used to extend the hybrid method to hydrocarbon species. Fig.20 demonstrates this approach for spectra collected in an ambient temperature mixture of $\mathrm{N}_{2}$ and $\mathrm{CH}_{4}$ using the spectrometer described in Ref. [20], with a low (1200 lines/mm, upper plot), and a high (4165 lines/mm, lower plot) dispersion grating. In the hybrid approach, "stick" spectra for all major species $\left(\mathrm{N}_{2}, \mathrm{O}_{2}, \mathrm{H}_{2}, \mathrm{H}_{2} \mathrm{O}, \mathrm{CO}_{2}\right.$ and $\mathrm{CO})$ are obtained from quantum mechanical calculations using Ramses [11], with no slit function. The stick spectra are then convolved with the instrument transfer function to generate spectra that match the instrument resolution. The instrument transfer function is provided by the Rayleigh scattering image of the laser profile in air, appropriately scaled by the magnification ratio between the Raman and Rayleigh imaging systems. For the hydrocarbons $\left(\mathrm{CH}_{4}, \mathrm{C}_{2} \mathrm{H}_{4}\right.$, $\mathrm{C}_{2} \mathrm{H}_{6}, \mathrm{CH}_{2} \mathrm{O}, \mathrm{C}_{3} \mathrm{H}_{8}$, and DME), synthetic spectra computed at the appropriate dispersion replace the stick spectra. The top portion of Fig. 20 compares measured, low-dispersion, ambient temperature, nitrogen and methane spectra (in blue) to the spectra (dotted red line) obtained by convolution of the "stick" spectrum of nitrogen and the synthetic spectrum of methane with the instrument transfer function. The lower portion of Fig. 20 shows (in blue) the methane, ambient temperature spectrum, collected with the high dispersion grating. Spectra collected with the high dispersion grating (high-dispersion spectra) have the same dispersion of the high resolution spectra discussed in this paper, but lower spectral resolution because no slit is used. The instrument function used to convolve the synthetic spectra for the high dispersion leg is $14 \%$ narrower than what used for the low dispersion spectra. The compression of the instrument function accounts for the broadening in the synthetic spectra introduced by spectrometer used to collect the high resolution spectra. This broadening can be neglected for the low dispersion spectra but it is a significant contribution to the high dispersion spectra. The $14 \%$ compression factor was optimized to minimize the residual (black curve). The plots show that the convolution from the synthetic spectra provides spectra that are as accurate as the ones obtained from Ramses, for both the low and the high dispersion spectrometer. The convolved spectra can then be integrated over the spectral regions associated with the Raman channels to evaluate the temperature and position dependent elements of the matrix $C$.

Unfortunately, the experimental and synthetic spectra are limited to $860 \mathrm{~K}$, but hydrocarbons are also present in flames at higher temperatures. Extrapolation or further experimental calibrations are needed to extend the synthetic spectra to higher temperature. Care must be taken when using the third order polynomial of Fig. 3 to extrapolate the temperature dependence to higher temperatures not reachable with the heater. The third order polynomial approximating the experimental $\mathrm{N}_{2}$ measurements provides results that are within $1 \%$ of the Ramses prediction up to $1700 \mathrm{~K}$, but $10 \%$ different at $2000 \mathrm{~K}$. This suggests that extrapolation up to $1500 \mathrm{~K}$ may provide reasonable results for the hydrocarbons Raman signal temperature dependence, but it is not possible to quantify the uncertainty associated with such extrapolation. Extrapolated signal of methane in the $\mathrm{CH}$-stretch region is within $3 \%$ of the experimental measurements taken in a laminar flame from a Tsuji burner.[13] The synthetic spectra can be extended to higher temperature by an iterative process. The amplitude and width of each Gaussian used to generate 
the synthetic spectra are expressed as a third order polynomial of the temperature and can be extrapolated to higher temperatures. The polynomials describing the amplitude of the Gaussian allow negative values at temperatures above $860 \mathrm{~K}$, but such values are unphysical and are replaced with values equal to $1 / 10^{\text {th }}$ of the smallest positive value. The ratio between the integrated signal predicted from the polynomials and the integrated signal from the extrapolated synthetic spectra is computed, and used to scale the peak amplitudes. Polynomials for the peak amplitudes are recomputed, and the process iterated until the change in the integrated signal is within $1 \%$ of its value. Synthetic methane spectra in the 300-1400 K range obtained with this process are shown in Fig. 21. We expect only a qualitative agreement with the actual Raman spectra at these temperatures, but the integrated signal matches the extrapolated value. Extending this approach to the other hydrocarbons requires experimental measurements in stable laminar flames, like the one provided by the Tsuji burner, so as to derive the Raman signal dependence at higher temperature. In the introduction we showed the benefits of a dual resolution Raman instrument, with a higher dispersion grating to image the $\mathrm{CH}$-stretch region. Measurements in a Tsuji burner, for different hydrocarbon-air flames, taken with the proposed dual-resolution Raman instrument, will provide the temperature dependence of the Raman signal at flame temperatures. Temperature dependence curve can be measured for the different Raman channels of the high resolution spectra, and the synthetic spectra will be extrapolated to match the signal in each channel, rather than in entire $\mathrm{CH}$-stretch region, providing more accurate results. These measurements require the development of the dual-resolution Raman spectrometer and are beyond the scope of the present paper.

\section{Conclusion}

Experimental Raman spectra of methane, ethane, ethylene, and DME, at temperatures ranging from $300 \mathrm{~K}$ to $860 \mathrm{~K}$ have been collected at higher resolution than what is generally used for combustion applications. The Raman spectra and their temperature dependence were reported and discussed, and the spectra are available in graphical and tabular form. Spectra of formaldehyde at high temperatures are also obtained from pyrolysis of DME. Synthetic spectra, generated as combinations of Gaussian curves with amplitude and width being functions of the temperature, were discussed and are provided in tabular form. Synthetic spectra for methane were extrapolated to higher temperature, with the constraint that the integrated signal satisfies the temperature dependence previously observed. Future work will focus on measurements in stable, laminar flames from a Tsuji burner to extend the synthetic spectra at higher temperatures for the remaining hydrocarbons, following the strategy adopted for methane. The synthetic spectra will enable application of the hybrid approach to Raman measurements of hydrocarbons-air flames with fuels more complex than methane. In particular, measurements with a dual-resolution Raman instrument, combined with the hybrid approach enabled by the Raman spectra here discussed, will enable simultaneous measurements of DME and its major combustion intermediates in DME-air flames.

\section{Acknowledgements}

Work was supported by the Division of Chemical Sciences, Geosciences and Biosciences, Office of Basic Energy Sciences, US Department of Energy. Additional support was provided by the Department of Energy NNSA under Grant No. DE-FC52-08NA28615. Sandia National Laboratories is a multiprogram laboratory operated by Sandia Corporation, a Lockheed Martin 
Company, for the United States Department of Energy under contract DE-AC04-94-AL85000. Contributions by Bob Harmon in support of these experiments are gratefully acknowledged.

\section{Appendix}

The file named "Experimental Raman spectra" contains in tabular form the experimental Raman spectra discussed in the paper. Each table (Excel sheet) contains the spectra of one hydrocarbon for one polarization (no polarizer, perpendicular, parallel). The first column (labelled "wn ( $\left.\mathrm{cm}^{-1}\right)$ ") is the Raman shift in wavenumber, the remaining columns are the spectra at the temperature indicated in the first row.

The file named "Coefficients for synthetic spectra" contains the parameters needed to generate the synthetic spectra. The synthetic spectra are obtained as :

$$
S(w, T)=\sum_{i=1}^{N} A_{i}(T) \exp \left(\left(w-\mu_{i}\right) \cdot 2 \sqrt{\ln 2} / b_{i}(T)\right)^{2}
$$

Where $S$ is the Raman signal, $w$ is the Raman shift, $T$ is the temperature. The parameters $A_{i}$ and $b_{i}$ are obtained as a third order and a first order polynomial of the temperature

$$
A_{i}(T)=a_{0}^{i}+a_{1}^{i} T+a_{2}^{i} T^{2}+a_{3}^{i} T^{3}
$$

And

$$
b_{i}(T)=b_{0}^{i}+b_{1}^{i} T
$$

The parameters $\mu_{\mathrm{i}}, a_{0}^{i}, a_{1}^{i}, a_{2}^{i}, a_{3}^{i}, b_{0}^{i}$ and $b_{1}^{i}$ are listed in the supplemental file "Coefficients for synthetic spectra" for the perpendicularly polarized Raman spectra discussed in the paper.

\section{Figures}




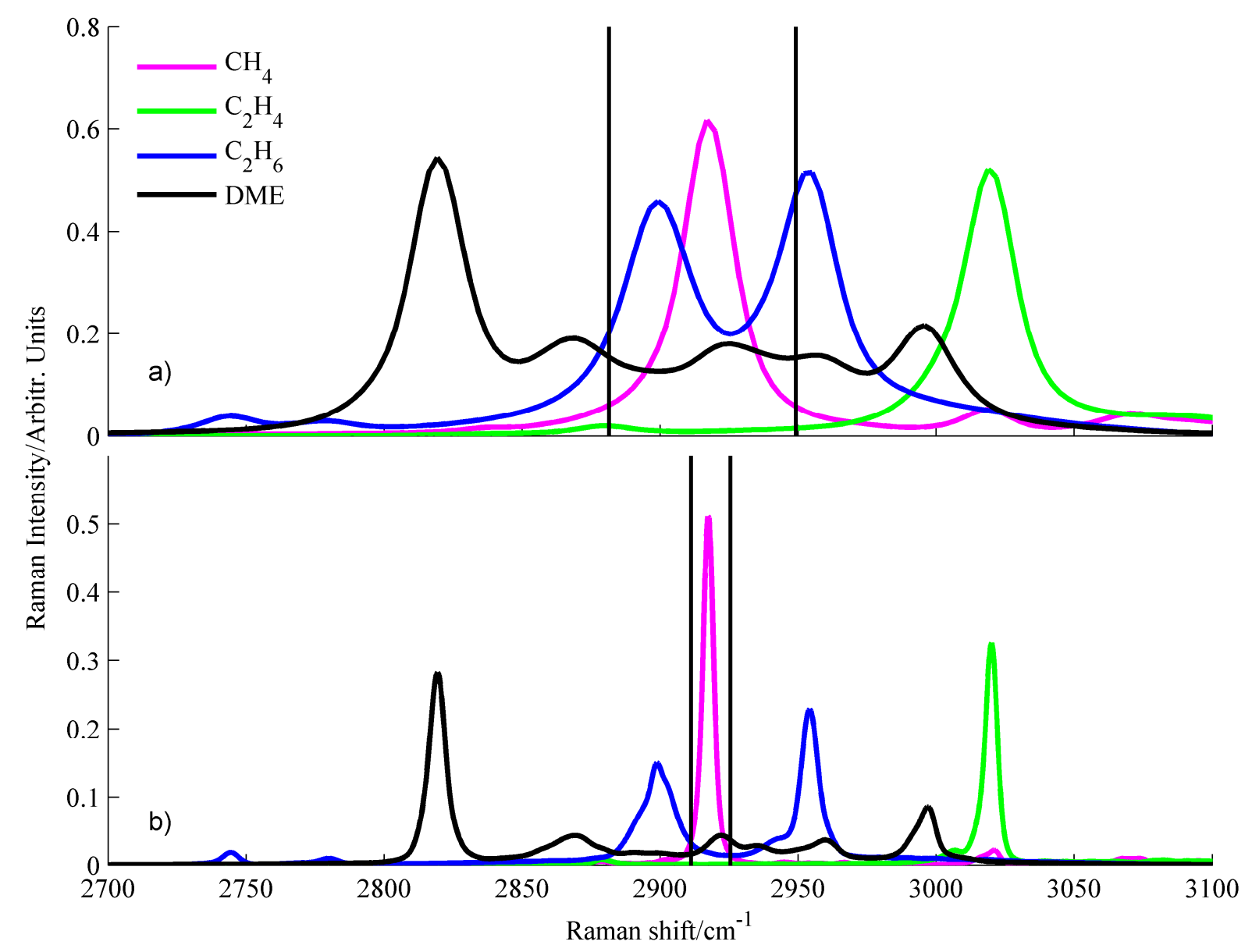

Figure 1. a). Raman spectra of methane, ethane, ethylene and DME in the CH-stretch region collected with the spectrometer described in Ref.1. Fig 1b) shows the same spectra collected with a higher dispersion grating. The vertical black lines define a plausible $\mathrm{CH}_{4}$ Raman channel. Note that the Raman channel is not the same spectral interval for low and high dispersion spectra. The horizontal axis is the same for both figures.
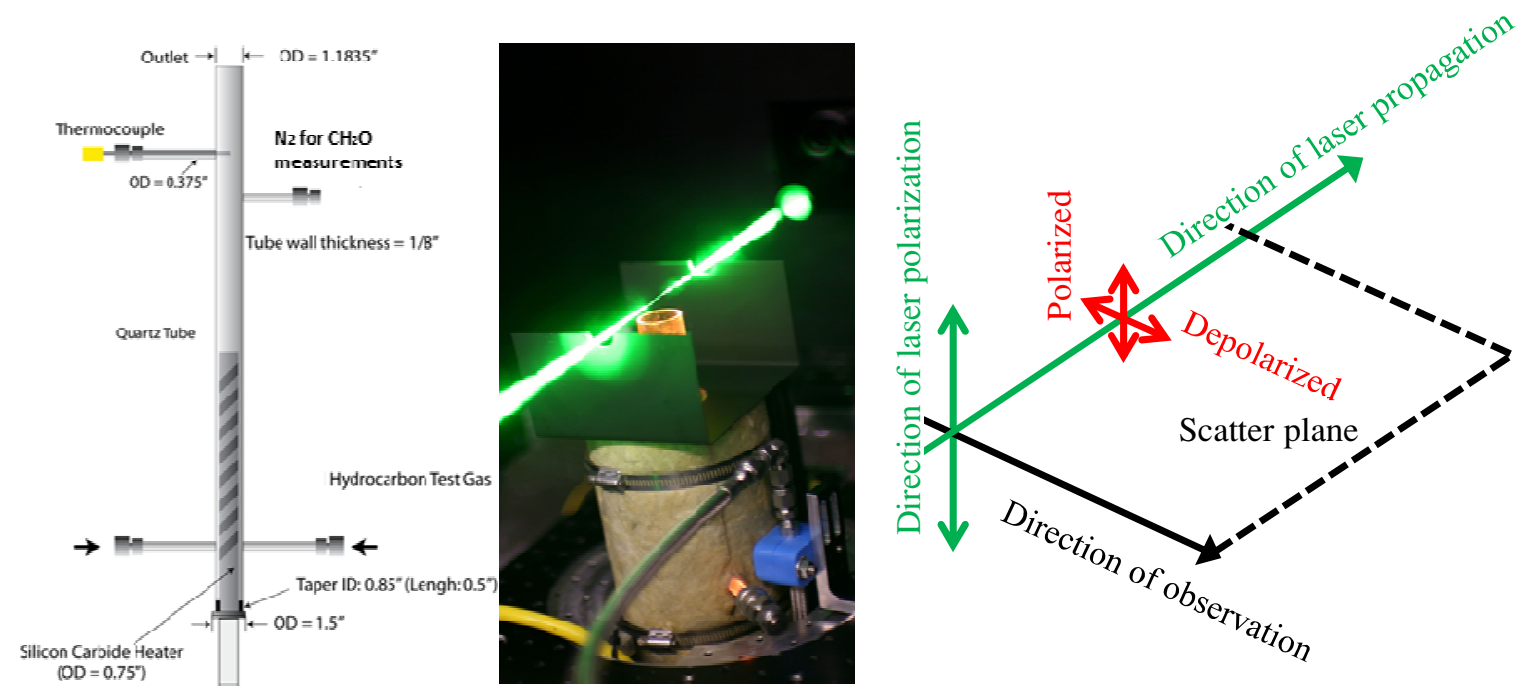

Figure 2. a) Schematic, b) photograph of the gas heater, and c) polarization diagram. 


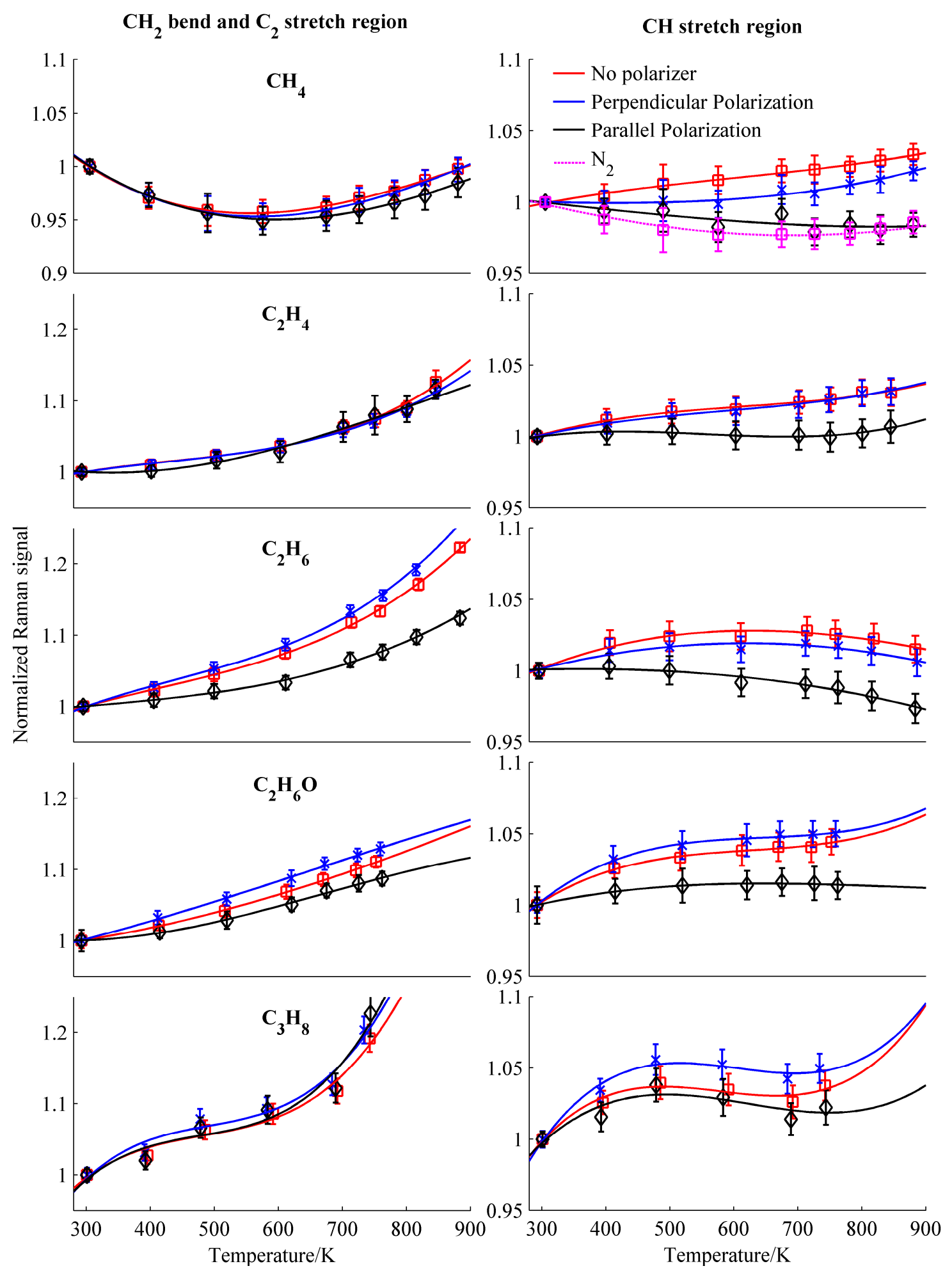

Figure 3. Temperature dependence of the normalized Raman cross section per molecule for various hydrocarbons in the $\mathrm{CH}_{2}$-bend (left column) and the $\mathrm{CH}$-stretch region (right column). Symbols are the average experimental results, the error bars represent plus or minus a standard deviation, and the continuous lines are a third order polynomial fit. The continuous line for $\mathrm{N}_{2}$ is obtained from Ramses [11]. 


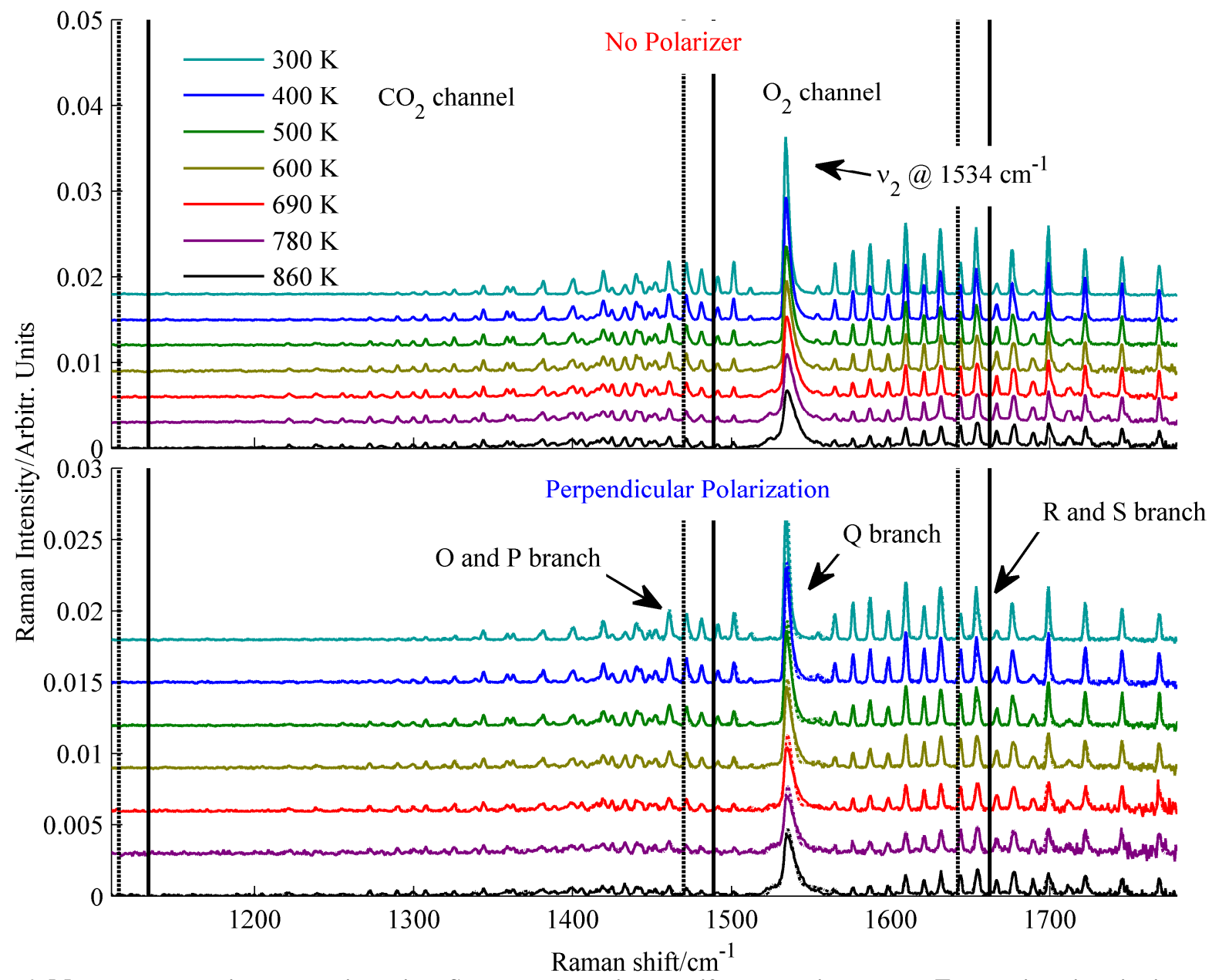

Figure 4. Methane spectra in the dyadic region. Spectra are vertically shifted to avoid overlap. The vertical lines indicate the spectral regions associated with the $\mathrm{CO}_{2}$ and $\mathrm{O}_{2}$ channels in the matrix inversion approach used for Raman combustion measurements. Dashed spectra are synthetic spectra. Dashed vertical lines define the spectra regions associated with the $\mathrm{CO}_{2}$ and $\mathrm{O}_{2}$ channels at the edge of the probe line.

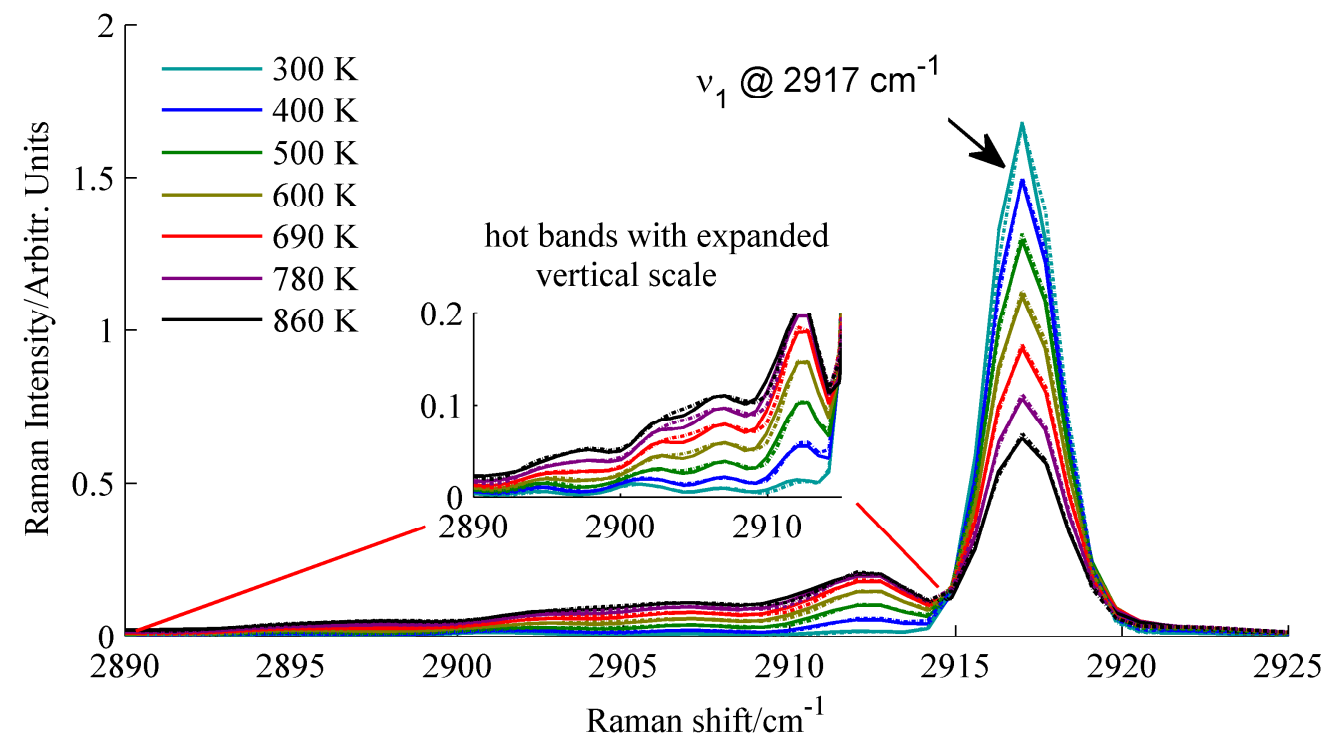

Figure 5. Methane spectra in the pentad region. The spectra are perpendicularly polarized. 


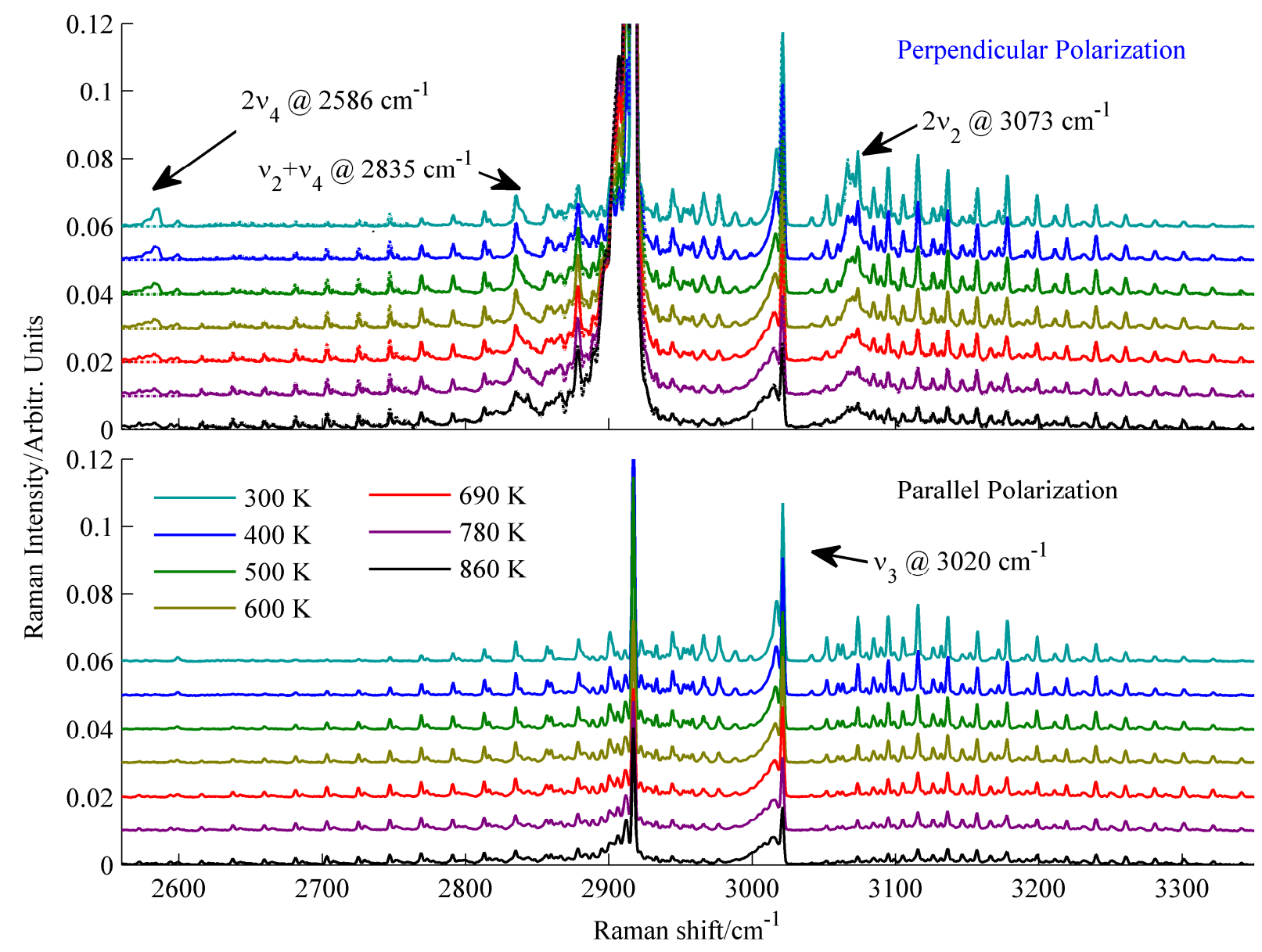

Figure 6. Close-up of methane spectra in the pentad region with extended horizontal and expanded vertical scales. The components of the spectra with polarization perpendicular (top figure) and parallel (bottom) to the excitation laser beam polarization are shown.

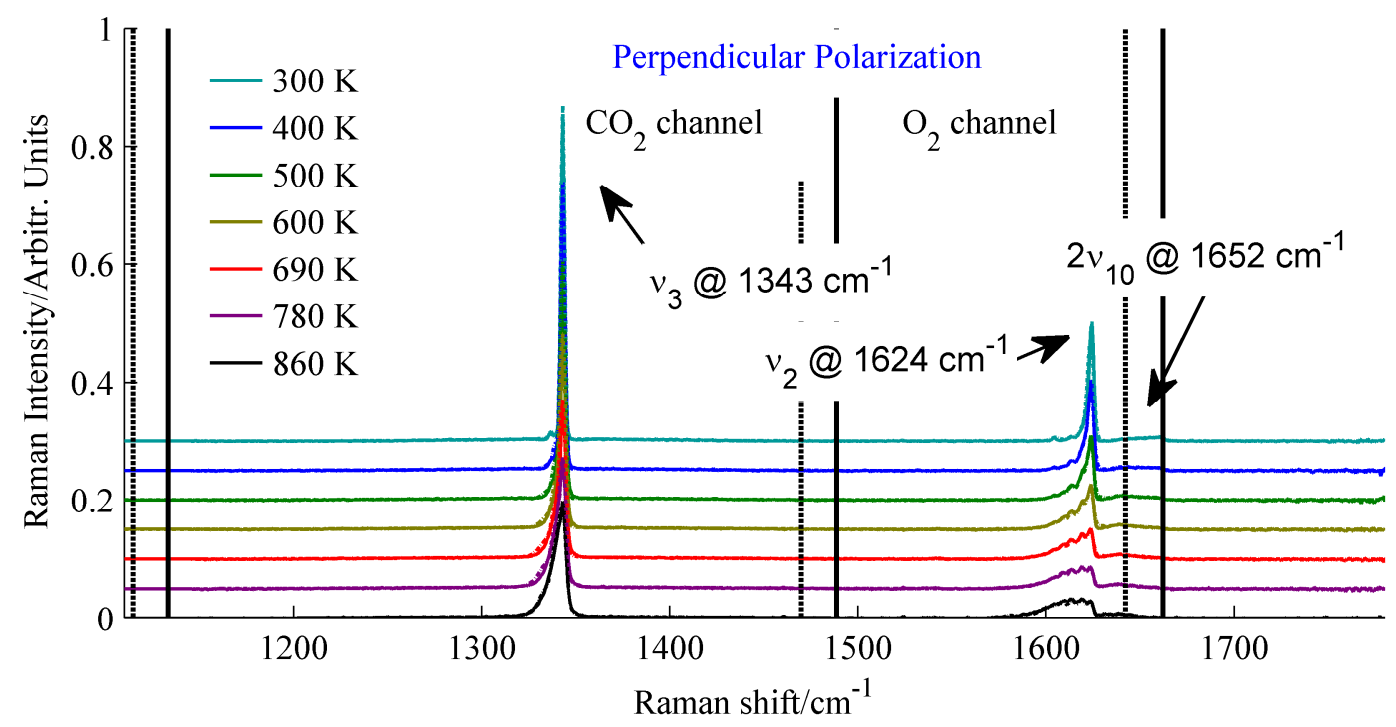

Figure 7. Ethylene spectra in the $1100-1780 \mathrm{~cm}^{-1}$ range. 


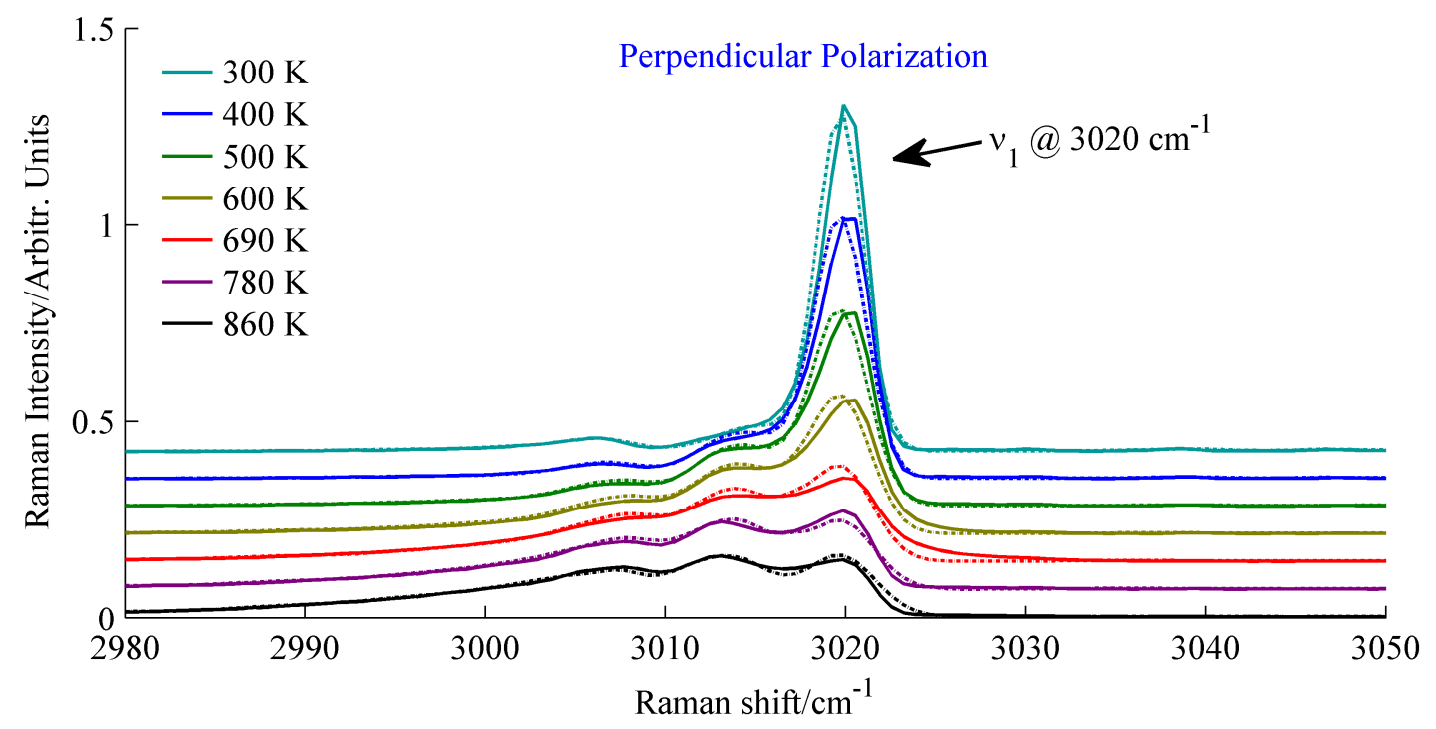

Figure 8. Ethylene spectra in the $2980-3050 \mathrm{~cm}^{-1}$ range.

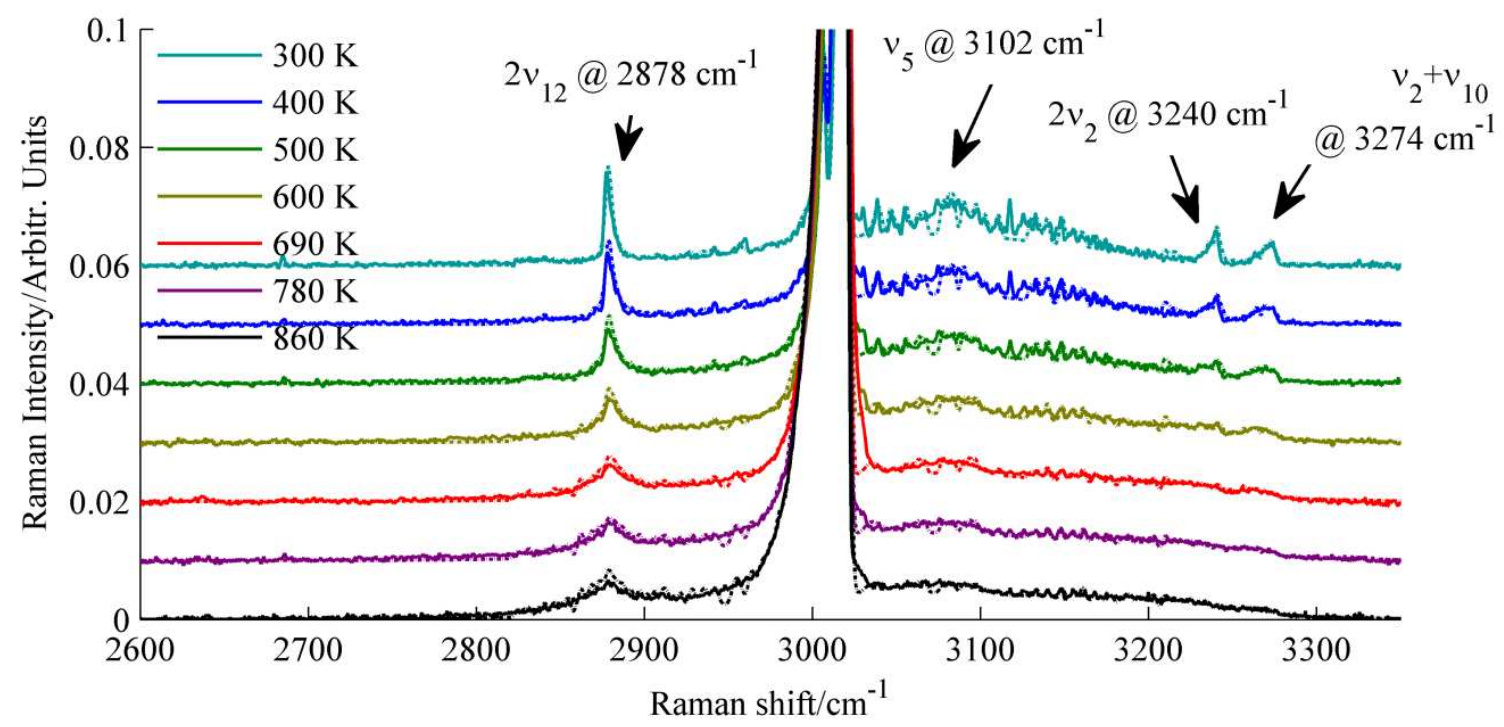

Figure 9.Close-up of the "perpendicular" component of the ethylene spectra in the $2600-3300 \mathrm{~cm}^{-1} \mathrm{range}^{\text {with }}$ extended horizontal and expanded vertical scales. 


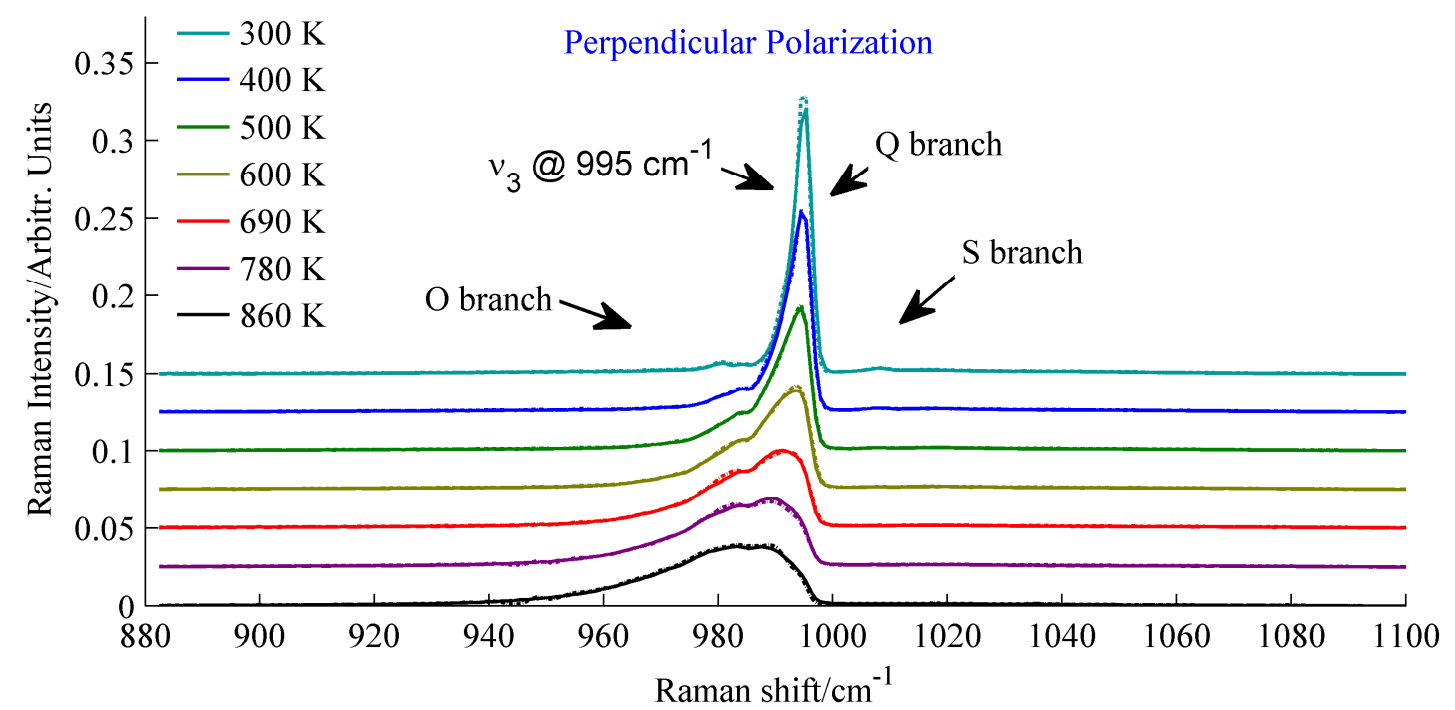

Figure 10. Ethane spectra in the $\mathrm{C}_{2}$ region $\left(880-1100 \mathrm{~cm}^{-1}\right)$.

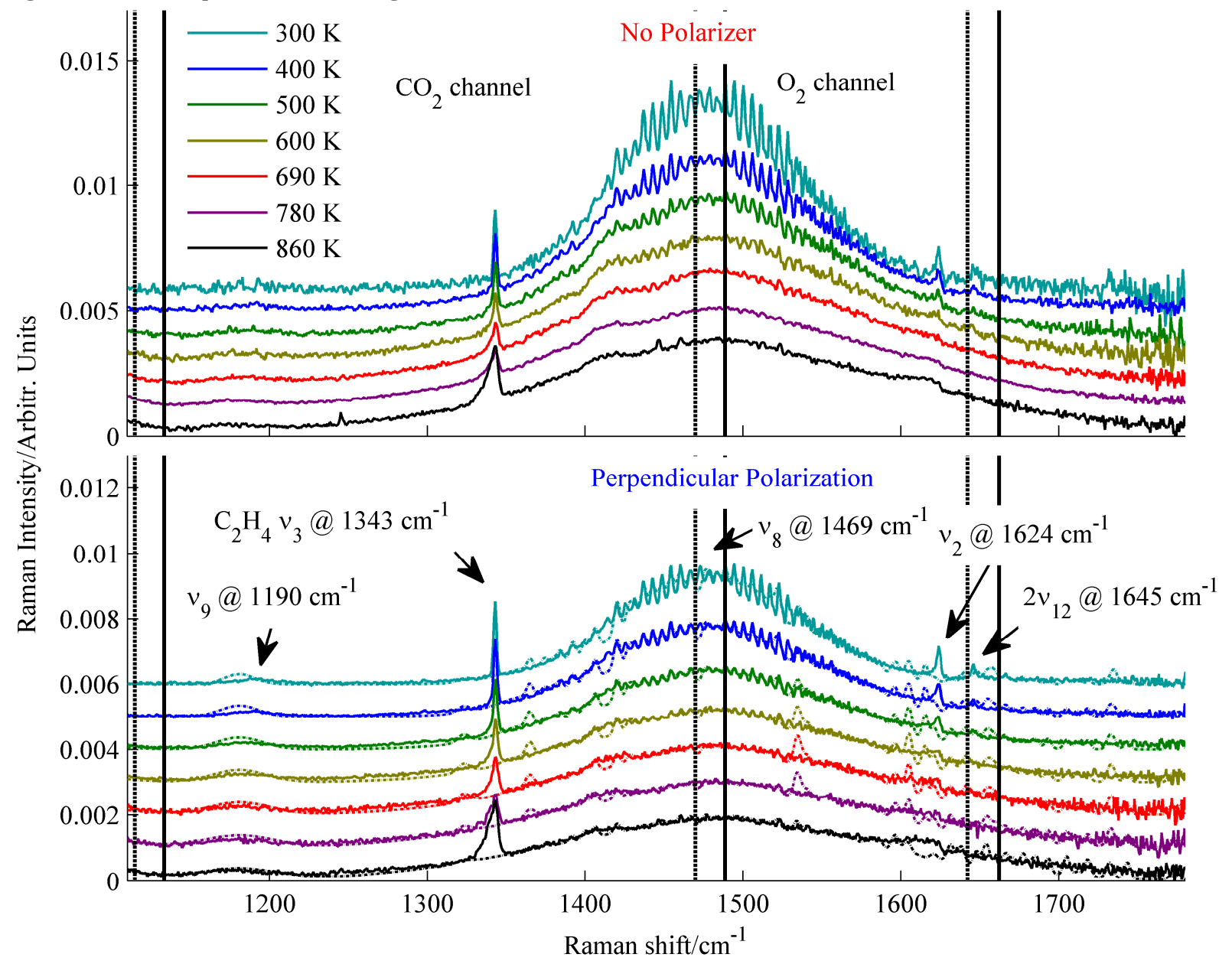

Figure 11. Ethane spectra in the $\mathrm{CO}_{2}$ and $\mathrm{O}_{2}$ region $\left(1100-1780 \mathrm{~cm}^{-1}\right)$. 


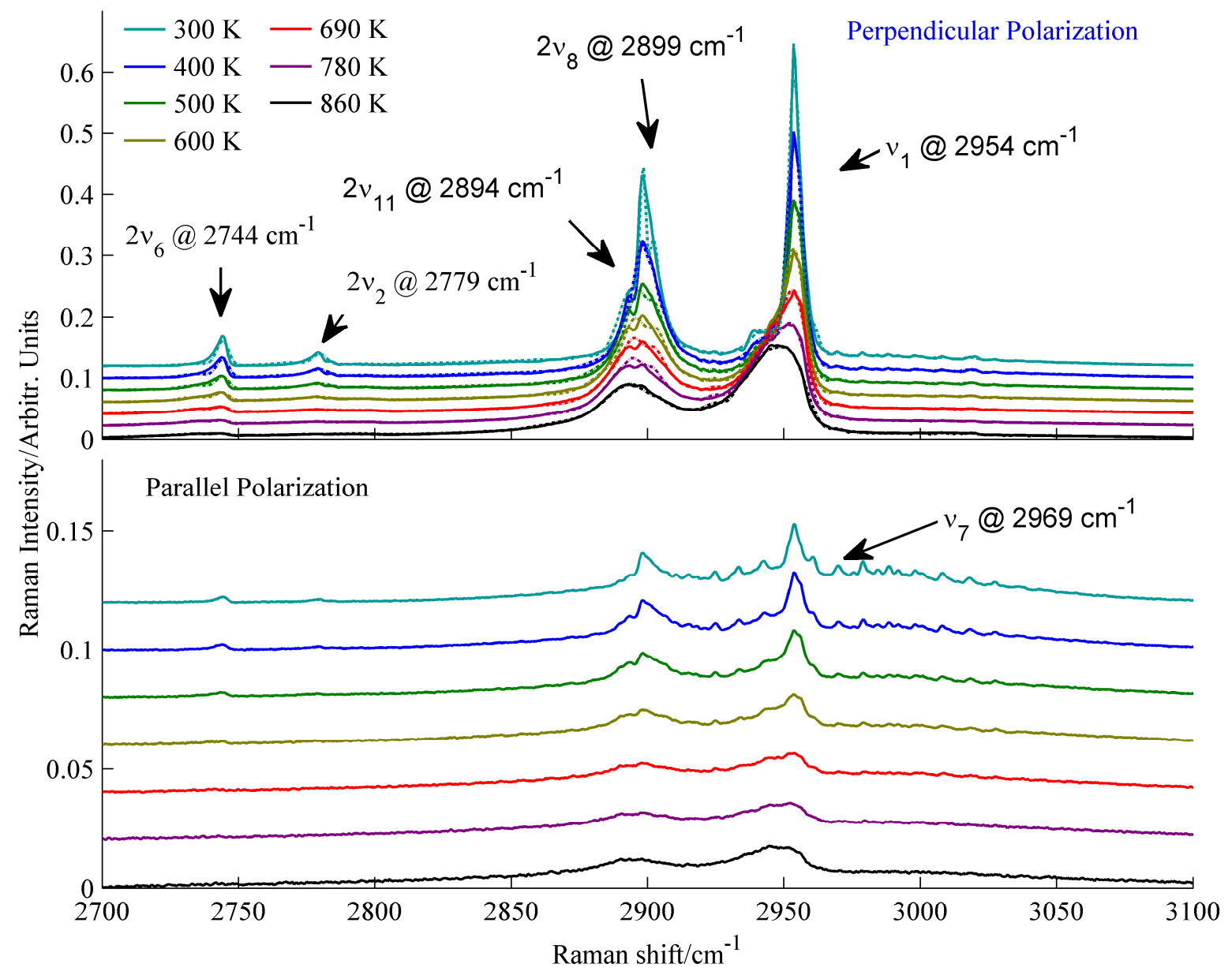

Figure 12. Ethane spectra in the $\mathrm{CH}$-stretch region $\left(2700-3100 \mathrm{~cm}^{-1}\right)$.

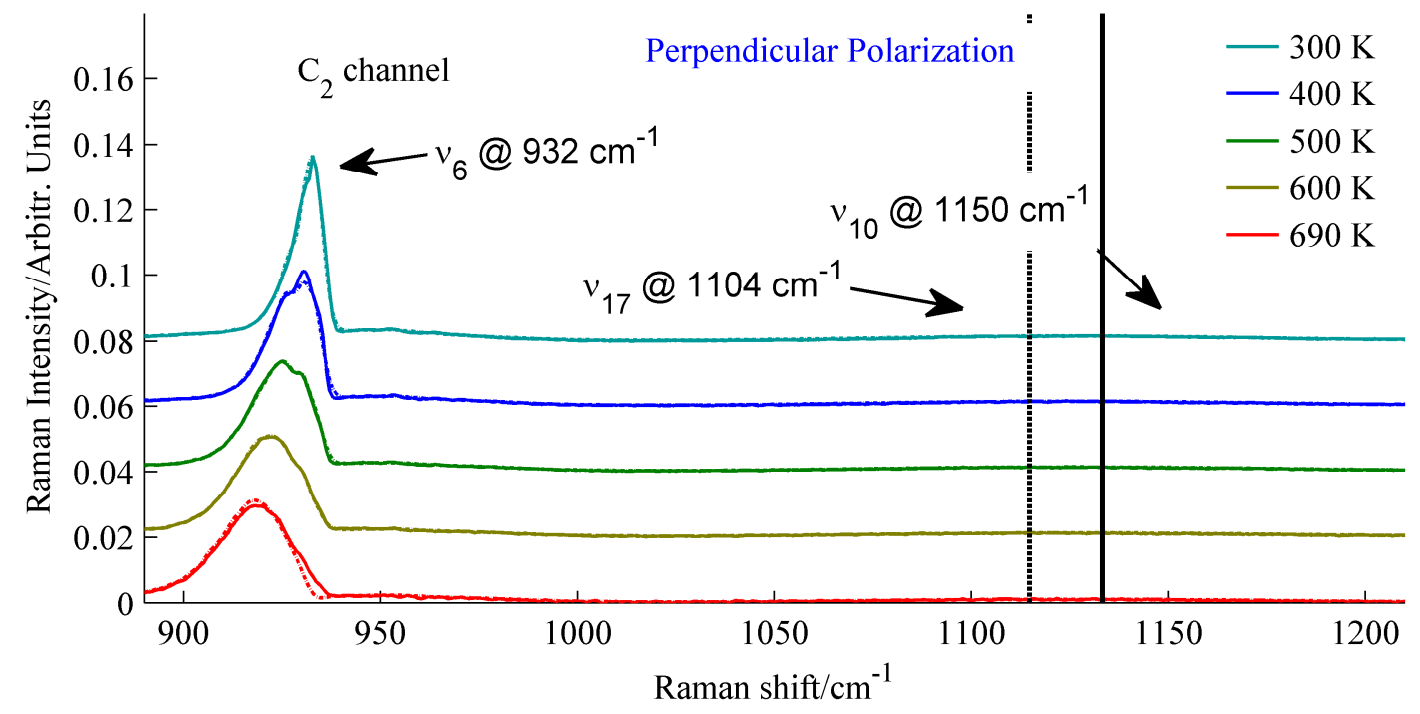

Figure 13. DME spectra in the $\mathrm{C}_{2}$ region $\left(880-1100 \mathrm{~cm}^{-1}\right)$. 


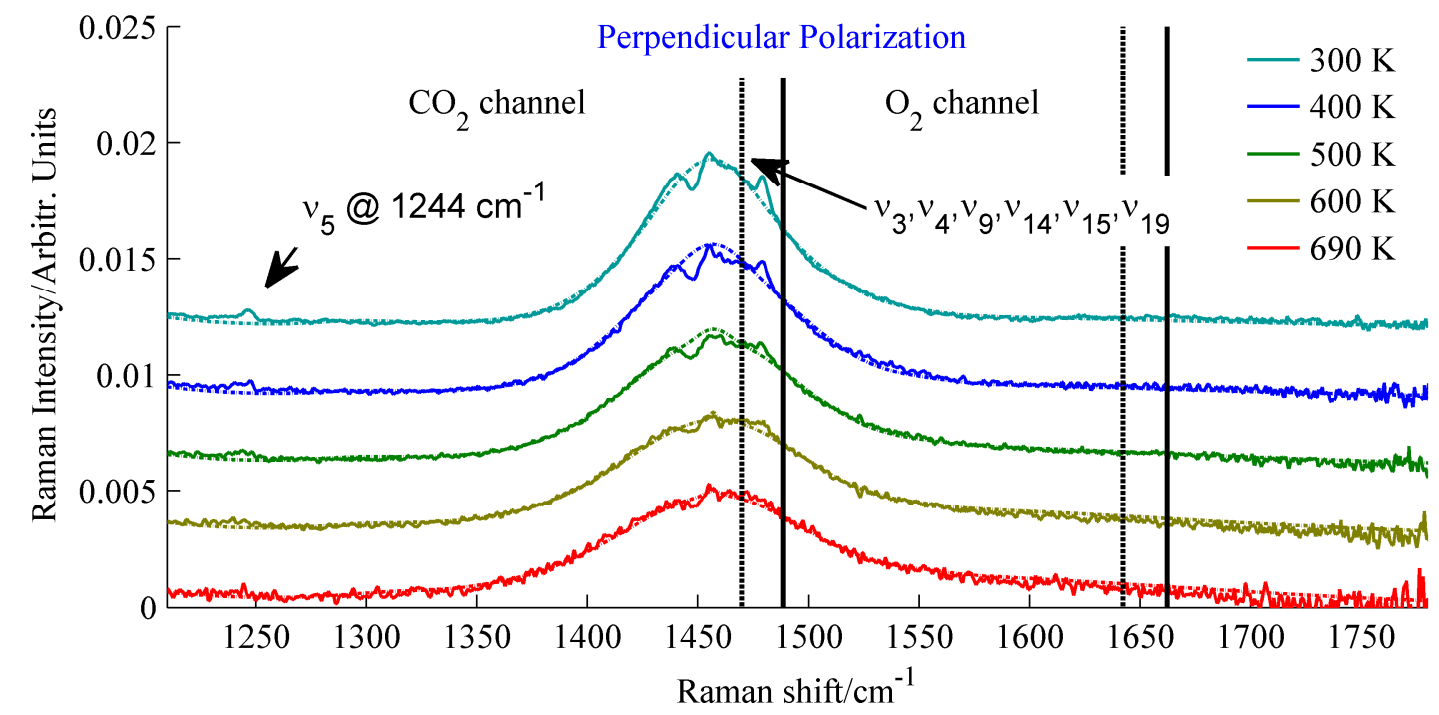

Figure 14. DME spectra in the $\mathrm{CO}_{2}$ and $\mathrm{O}_{2}$ region $\left(1100-1780 \mathrm{~cm}^{-1}\right)$.

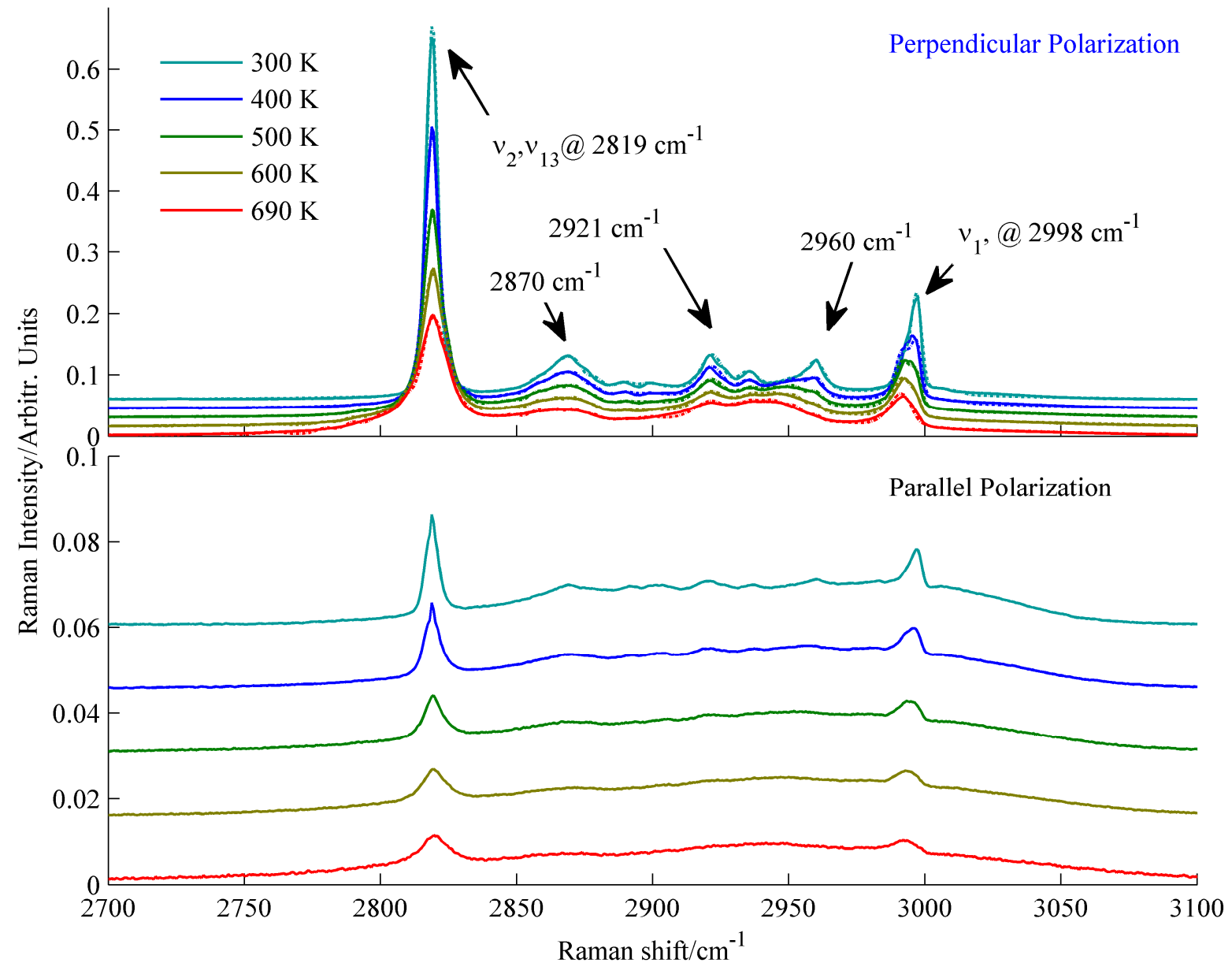

Figure 15. DME spectra in the $\mathrm{CH}$-stretch region $\left(2700-3100 \mathrm{~cm}^{-1}\right)$. 


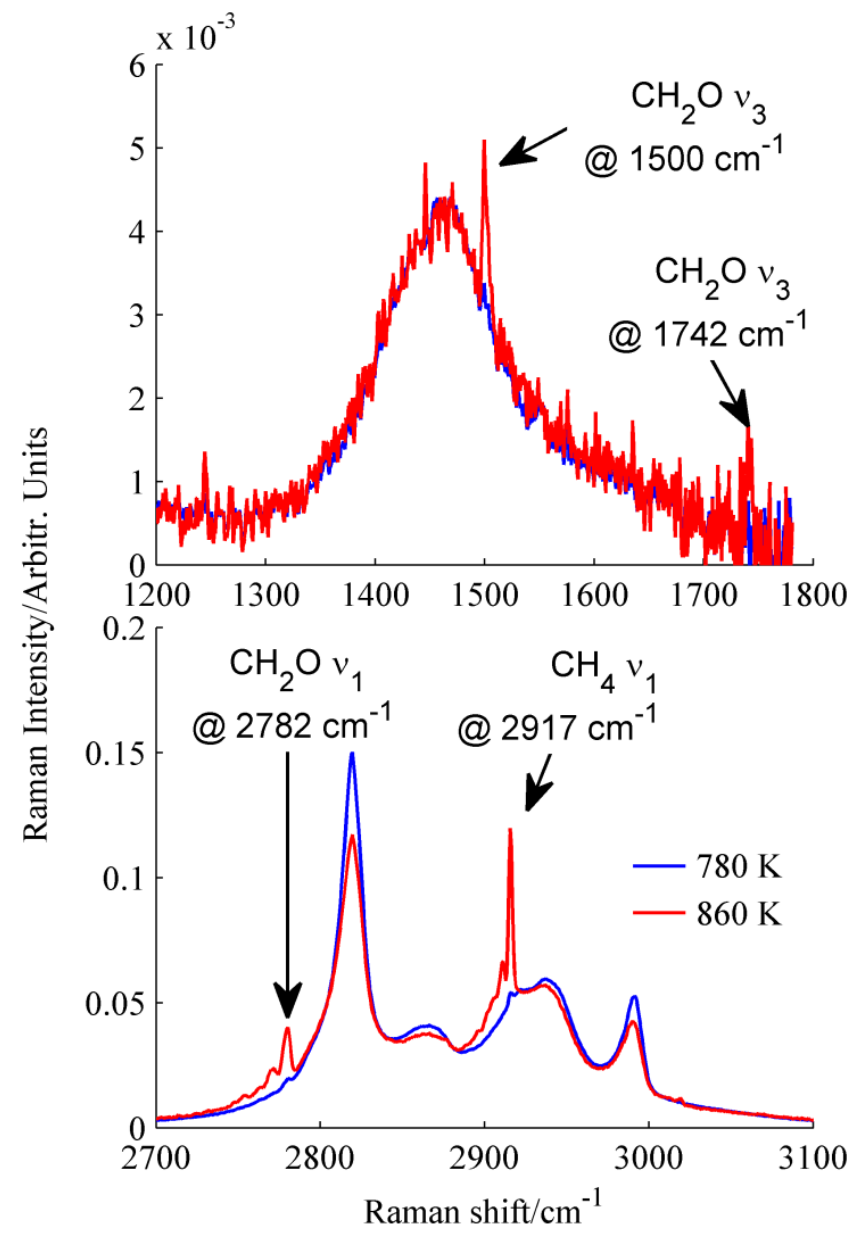

Figure 16. Raman spectra collected in DME at 780 and $860 \mathrm{~K}$. The $v_{1} \mathrm{CH}$-stretch mode of methane and the $v_{1}, v_{2}, v_{3}$ vibrational modes of formaldehyde are evident.

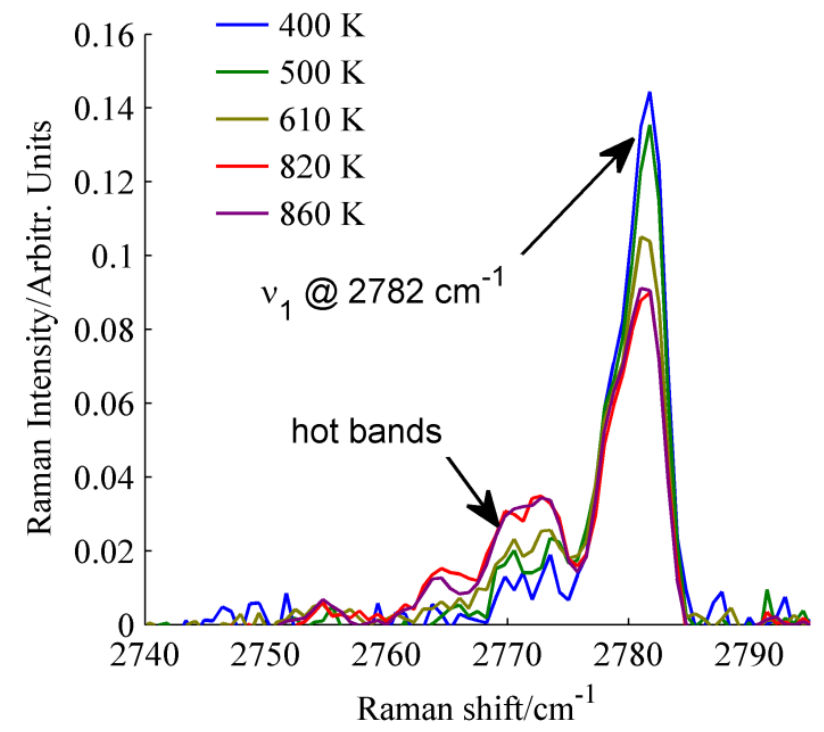

Figure 17. Raman spectra of the $v_{1}$ vibrational mode of formaldehyde. 


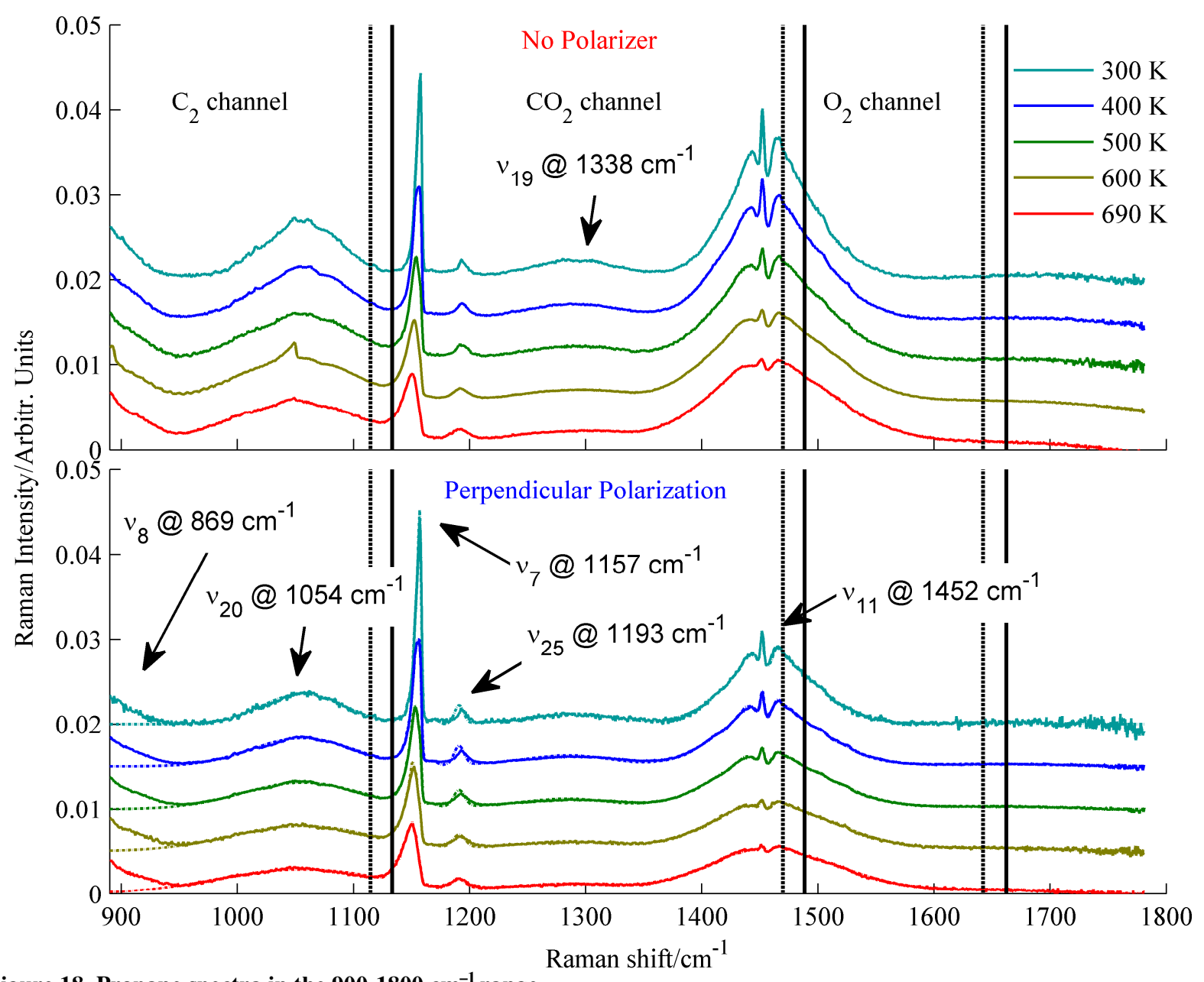

Figure 18. Propane spectra in the $900-1800 \mathrm{~cm}^{-1}$ range. 


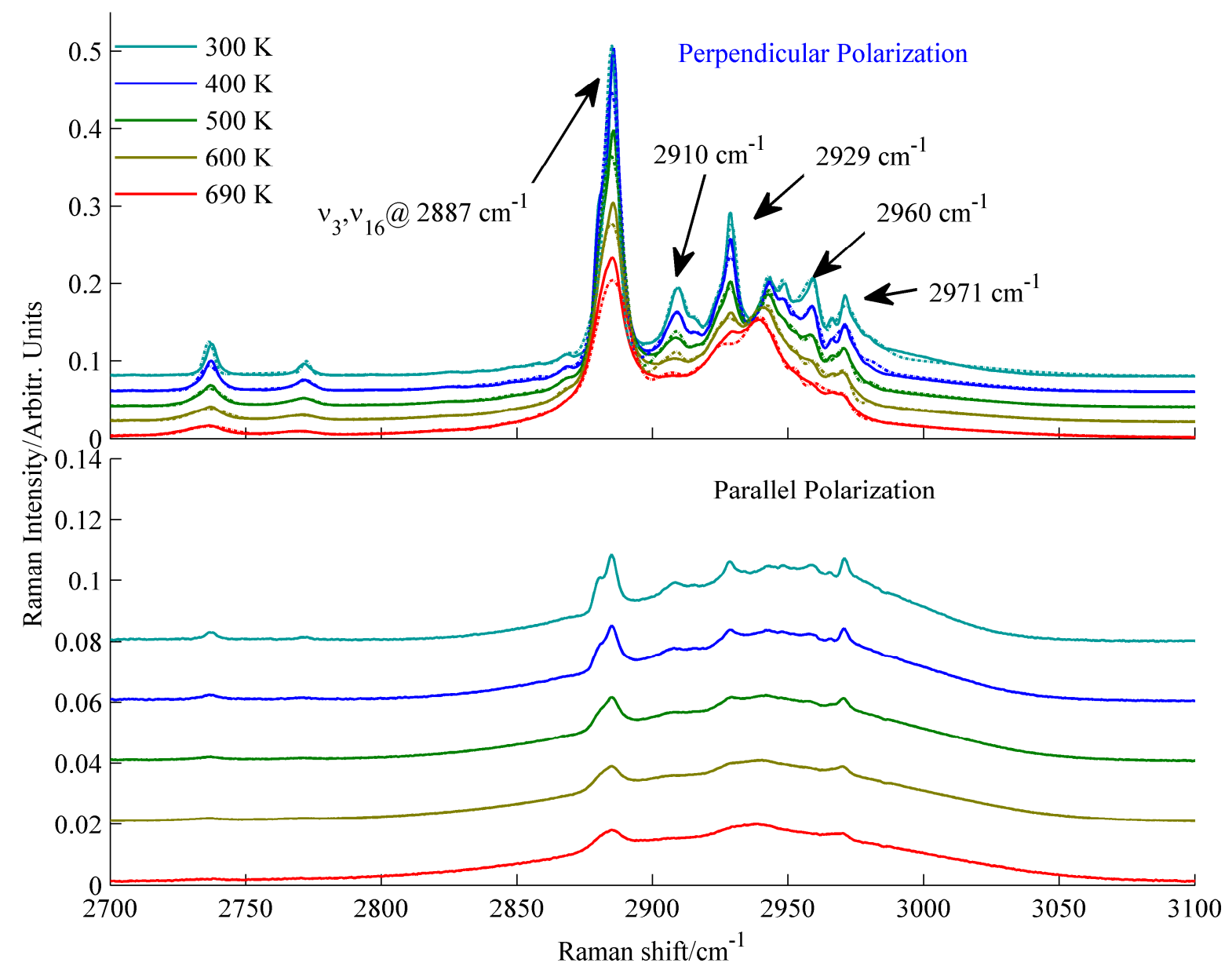

Figure 19. Propane spectra in the $\mathrm{CH}$-stretch region $\left(2700-3100 \mathrm{~cm}^{-1}\right)$. 

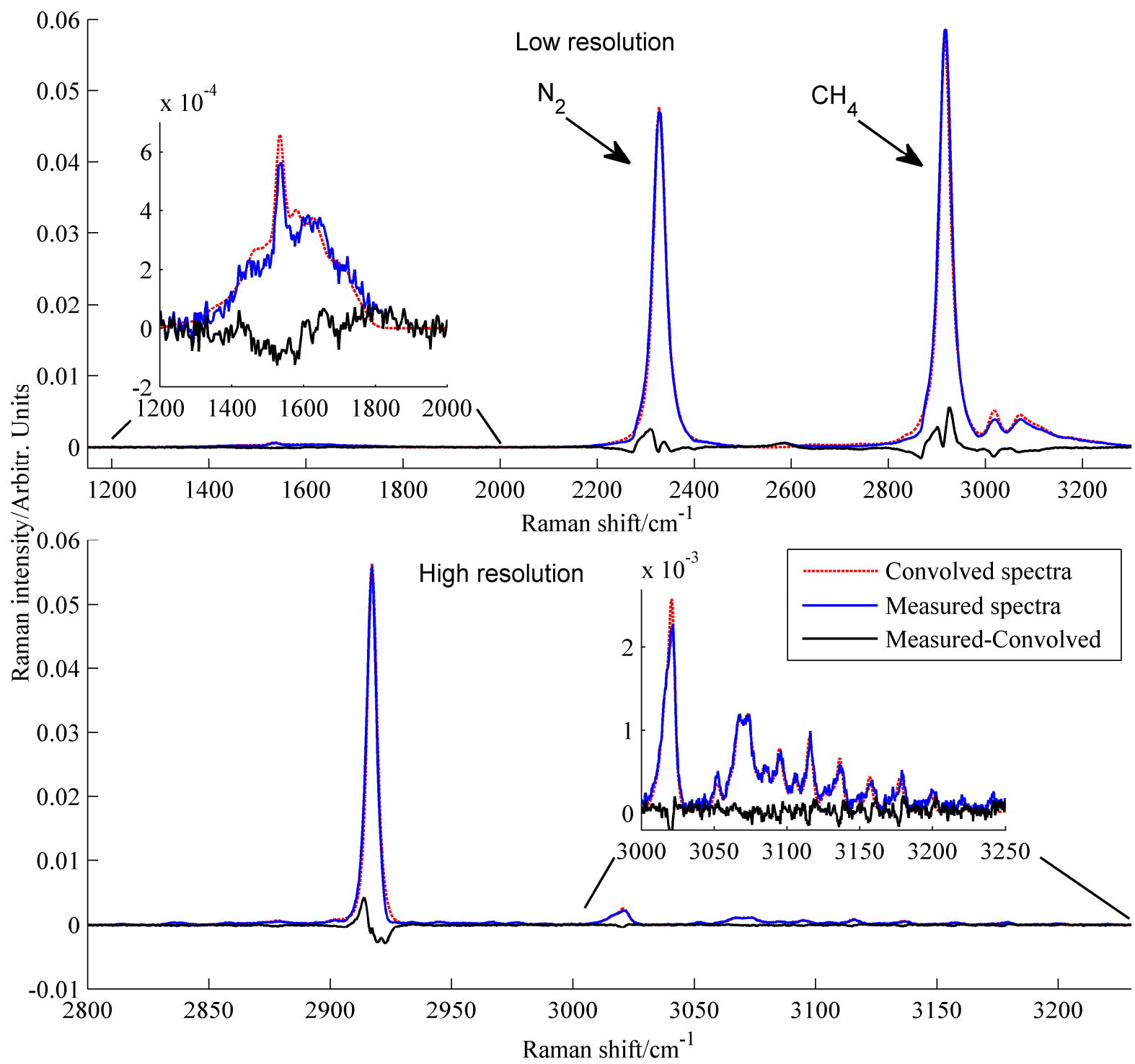

Figure 20. Measured (blue curve), convolved (red dotted curve), and residual (black curve) for nitrogen and methane at ambient temperature collected with a "low" and a " high" dispersion spectrometer. 


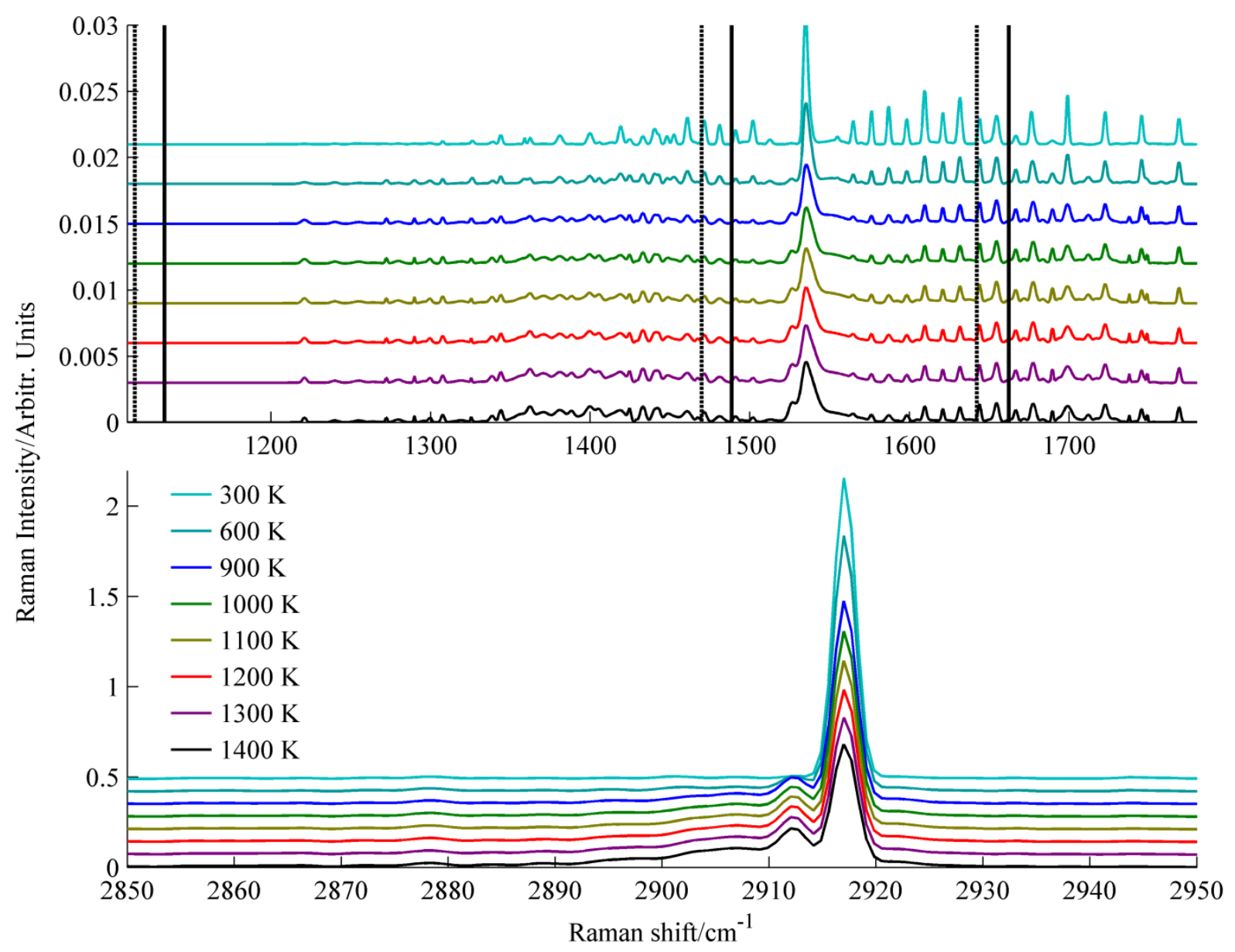

Figure 21. Synthetic spectra of methane from $300 \mathrm{~K}$ to $1400 \mathrm{~K}$.

Table 1. Integrated Raman signal and Rayleigh cross section ratioed to $\mathrm{N}_{2}$ taken without a polarizer

\begin{tabular}{|c|c|c|c|c|c|c|c|}
\hline & \multicolumn{3}{|c|}{ CH-stretch region $\left(2548-3434 \mathrm{~cm}^{-1}\right)$} & \multicolumn{3}{|c|}{$\mathrm{CH}_{2}$-bend region $\left(882-1791 \mathrm{~cm}^{-1}\right)$} & \multirow{2}{*}{$\begin{array}{c}\text { Rayleigh cross } \\
\text { section }\end{array}$} \\
\hline & $\begin{array}{c}\text { No } \\
\text { polarizer }\end{array}$ & $\begin{array}{l}\text { Perpendicular } \\
\text { Polarization }\end{array}$ & $\begin{array}{l}\text { Parallel } \\
\text { Polarization }\end{array}$ & $\begin{array}{c}\text { No } \\
\text { polarizer }\end{array}$ & $\begin{array}{l}\text { Perpendicular } \\
\text { Polarization }\end{array}$ & $\begin{array}{l}\text { Parallel } \\
\text { Polarization }\end{array}$ & \\
\hline $\mathrm{CH}_{4}$ & 11.63 & 8.98 & 1.89 & 0.58 & 0.30 & 0.21 & 2.16 \\
\hline $\mathrm{C}_{2} \mathrm{H}_{4}$ & 10.77 & 7.81 & 2.17 & 5.44 & 4.07 & 0.74 & 5.99 \\
\hline $\mathbf{C}_{2} \mathbf{H}_{6}$ & 19.07 & 14.45 & 3.32 & 3.16 & 1.95 & 0.84 & 6.46 \\
\hline $\mathrm{C}_{2} \mathrm{H}_{6} \mathrm{O}$ & 21.04 & 16.01 & 3.61 & 3.81 & 2.16 & 1.22 & 9.37 \\
\hline $\mathrm{C}_{3} \mathrm{H}_{8}$ & 26.44 & 20.13 & 4.67 & 3.62 & 2 & 1.24 & 13.58 \\
\hline
\end{tabular}

\section{References}

[1] Eckbreth AC. Laser Diagnostics for Combustion Temperature and Species. New York: Gordon and Breach Publishers; 1996.

[2] Nguyen QV, Dibble RW, Carter CD, Fiechtner GJ, Barlow RS. Raman-LIF measurements of temperature, major species, $\mathrm{OH}$, and $\mathrm{NO}$ in a methane-air bunsen flame. Combustion and Flame. 1996;105:499-510.

[3] Barlow RS, Frank JH. Effects of turbulence on species mass fractions in methane/air jet flames. Symposium (International) on Combustion. 1998;27:1087-95. 
[4] Jun K, Quang-Viet N. Single-shot rotational Raman thermometry for turbulent flames using a lowresolution bandwidth technique. Measurement Science and Technology. 2008;19:015406.

[5] Fernández JM, Punge A, Tejeda G, Montero S. Quantitative diagnostics of a methane/air mini-flame by Raman spectroscopy. Journal of Raman Spectroscopy. 2006;37:175-82.

[6] Magnotti G, Barlow RS. Effects of high shear on the structure and thickness of turbulent premixed methane/air flames stabilized on a bluff-body burner. Combustion and Flame. 2014;submitted on March 2014.

[7] Sweeney MS, Hochgreb S, Barlow RS. The structure of premixed and stratified low turbulence flames. Combustion and Flame. 2011;158:935-48.

[8] Meares S, Prasad VN, Magnotti G, Barlow RS, Masri AR. Stabilization of piloted turbulent flames with inhomogeneous inlets. Proceedings of the Combustion Institute.

[9] Fuest F, Barlow RS, Geyer D, Seffrin F, Dreizler A. A hybrid method for data evaluation in 1-D Raman spectroscopy. Proceedings of the Combustion Institute. 2011;33:815-22.

[10] Dibble RW, Stårner SH, Masri AR, Barlow RS. An improved method of data aquisition and reduction for laser raman-rayleigh and fluorescence scattering from Multispecies. Appl Phys B. 1990;51:39-43.

[11] Geyer D. 1D-Raman/Rayleigh Experiments in a Turbulent Opposed-Jet: Technical University of Darmstadt, Germany; 2005.

[12] Zhao J. Image Curvature Correction and Cosmic Removal for High-Throughput Dispersive Raman Spectroscopy. Applied Spectroscopy. 2003;57:1368-75.

[13] Barlow RS, Karpetis AN, Frank JH, Chen JY. Scalar profiles and NO formation in laminar opposedflow partially premixed methane/air flames. Combustion and Flame. 2001;127:2102-18.

[14] Pellet GL, Vaden SN, Wilson LG. Opposed Jet Burner Extinction Limits: Simple Mixed Hydrocarbon Scramjet Fuels vs Air. In: AIAA, editor. 43rd AIAA/ASME/SAE/ASEE Joint Propulsion Conference \& Exhibit. Cincinnati2007.

[15] Fuest F, Barlow RS, Chen J-Y, Dreizler A. Raman/Rayleigh scattering and CO-LIF measurements in laminar and turbulent jet flames of dimethyl ether. Combustion and Flame. 2012;159:2533-62.

[16] Magnotti G, Geyer D, Barlow RS. Interference free spontaneous Raman spectroscopy for measurements in rich hydrocarbon flames. Proceedings of the Combustion Institute.

[17] Jourdanneau E, Chaussard F, Saint-Loup R, Gabard T, Berger H. The methane Raman spectrum from 1200 to 5500\&\#xa0;cm-1: A first step toward temperature diagnostic using methane as a probe molecule in combustion systems. Journal of Molecular Spectroscopy. 2005;233:219-30.

[18] Ekoto I, Barlow RS. Development of a Raman Spectroscopy Technique to Detect Alternate Transportation Fuel Hydrocarbon Intermediates in Complex Combustion Environments. In: REPORT S, editor. SANDIA REPORT: Sandia National Laboratories; 2012.

[19] James TM, Schlösser M, Fischer S, Sturm M, Bornschein B, Lewis RJ, et al. Accurate depolarization ratio measurements for all diatomic hydrogen isotopologues. Journal of Raman Spectroscopy. 2013;44:857-65.

[20] Barlow RS, Dunn MJ, Sweeney MS, Hochgreb S. Effects of preferential transport in turbulent bluffbody-stabilized lean premixed CH4/air flames. Combustion and Flame. 2012;159:2563-75.

[21] Utsav KC, Varghese PL. Accurate temperature measurements in flames with high spatial resolution using Stokes Raman scattering from nitrogen in a multiple-pass cell. Appl Opt. 2013;52:5007-21.

[22] Shimanouchi T. Tables of Molecular Vibrational Frequencies Consolidated Volume I: NSRDS NBS-39; 1972.

[23] O'Haver T. Peak Finding and Measurement. http://terpconnect.umd.edu/ toh/spectrum/PeakFindingandMeasurement.htm. 2008

[24] Thomas MA, Welsh HL. THE RAMAN SPECTRUM OF METHANE. Canadian Journal of Physics. 1960;38:1291-303. 
[25] Herranz J, Stoicheff BP. High-resolution Raman spectroscopy of gases: Part XVI. The v3 Raman band of methane. Journal of Molecular Spectroscopy. 1963;10:448-83.

[26] Hilico JC, Champion JP, Toumi S, Tyuterev VG, Tashkun SA. New Analysis of the Pentad System of Methane and Prediction of the (Pentad-Pentad) Spectrum. Journal of Molecular Spectroscopy. 1994;168:455-76.

[27] Hilico JC, Robert O, Loëte M, Toumi S, Pine AS, Brown LR. Analysis of the Interacting Octad System of 12CH4. Journal of Molecular Spectroscopy. 2001;208:1-13.

[28] Foster RB, Hills GW, Jones WJ. Raman spectra of asymmetric top molecules. Molecular Physics. 1977;33:1589-610.

[29] Knippers W, Van Helvoort K, Stolte S, Reuss J. Raman overtone spectroscopy of ethylene. Chemical Physics. 1985;98:1-6.

[30] Bermejo D, Cané E, Di Lonardo G, Luis Domenech J, Escribano R, Martínez RZ, et al. The v2, v3 and 2v10 Raman bands of ethylene (12C2H4). Molecular Physics. 2004;102:1659-69.

[31] Oomens J, Reuss J, Mellau GC, Klee S, Gulaczyk I, Fayt A. The Ethylene Hot Band Spectrum near $3000 \mathrm{~cm}-1$. Journal of Molecular Spectroscopy. 1996;180:236-48.

[32] Beyrau F, Seeger T, Malarski A, Leipertz A. Determination of temperatures and fuel/air ratios in an ethene-air flame by dual-pump CARS. Journal of Raman Spectroscopy. 2003;34:946-51.

[33] Van Helvoort K, Knippers W, Fantoni R, Stolte S. The Raman spectrum of ethane from 600 to 6500 cm-1 stokes shifts. Chemical Physics. 1987;111:445-65.

[34] Crawford BL, Avery WH, Linnett JW. Infra - Red and Raman Spectra of Polyatomic Molecules. III. Ethane. The Journal of Chemical Physics. 1938;6:682-5.

[35] Lepard DW, Sweeney DMC, Welsh HL. THE RAMAN SPECTRUM OF ETHANE-d6. Canadian Journal of Physics. 1962;40:1567-82.

[36] Lepard DW, Shaw DE, Welsh HL. THE v10 AND v11 RAMAN BANDS OF GASEOUS ETHANE. Canadian Journal of Physics. 1966;44:2353-62.

[37] Shaw DE, Welsh HL. THE RAMAN SPECTRUM OF ETHANE-1,1,1-d3. Canadian Journal of Physics. 1967;45:3823-35.

[38] Taylor RC, Vidale GL. Raman Spectrum and Vibrational Assignments of Gaseous Dimethyl Ether. The Journal of Chemical Physics. 1957;26:122-3.

[39] Allan A, McKean DC, Perchard JP, Josien ML. Vibrational spectra of crystalline dimethyl ethers. Spectrochimica Acta Part A: Molecular Spectroscopy. 1971;27:1409-37.

[40] Andersson Ö, Neij H, Bood J, Axelsson B, Alden M. Optical Characterization of Dimethyl Ether (DME) for Laser-based Combustion Diagnostics. Combustion Science and Technology. 1998;137:299-322.

[41] Coudert LH, Çarçabal P, Chevalier M, Broquier M, Hepp M, Herman M. High-Resolution Analysis of the v6, v17, and v21 Bands of Dimethyl Ether. Journal of Molecular Spectroscopy. 2002;212:203-7.

[42] Blom CE, Altona C, Oskam A. Application of self-consistent-field ab initio calculations to organic molecules. Molecular Physics. 1977;34:557-71.

[43] NIST. Dymethil ether. http://webbook.nist.gov/cgi/cbook.cgi?ID=C115106\&Mask=800. 2011

[44] Good DA, Francisco JS. Structure and Vibrational Spectra of Chlorofluorocarbon Substitutes: An Experimental and ab Initio Study of Fluorinated Ethers CHF2OCF3 (E125), CHF2OCHF2 (E134), and CH3OCF3 (E143A). The Journal of Physical Chemistry A. 1998;102:1854-64.

[45] Chapput A, Roussel B, Fleury G. Étude par spectroscopie Raman en phase vapeur des trois espèces deutérées du formaldéhyde. Journal of Raman Spectroscopy. 1973;1:507-11.

[46] Davidson DW, Stoicheff BP, Bernstein HJ. The Infrared and Raman Spectra of Formaldehyde - d1 Vapor. The Journal of Chemical Physics. 1954;22:289-94.

[47] Flurry Jr RL. Vibrational assignments for propane from the nonrigid molecular symmetry group. Journal of Molecular Spectroscopy. 1975;56:88-92. 